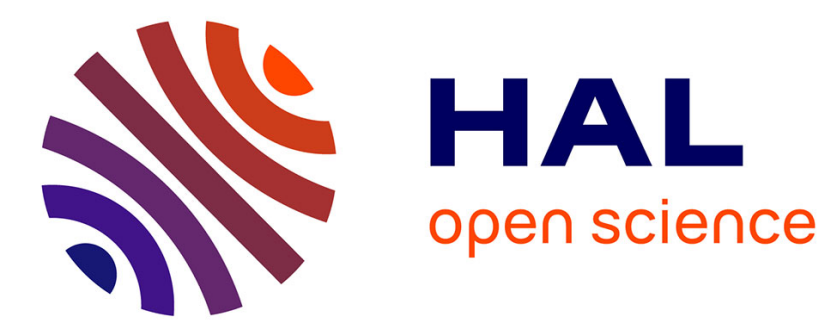

\title{
A fresh look at dense hydrogen under pressure. I. An introduction to the problem, and an index probing equalization of $\mathbf{H}-\mathbf{H}$ distances
}

Vanessa Labet, Paulina Gonzalez-Morelos, Roald Hoffmann, N W Ashcroft

\section{- To cite this version:}

Vanessa Labet, Paulina Gonzalez-Morelos, Roald Hoffmann, N W Ashcroft. A fresh look at dense hydrogen under pressure. I. An introduction to the problem, and an index probing equalization of H-H distances. Journal of Chemical Physics, 2012, 136, 10.1063/1.3679662 . hal-02202225

\section{HAL Id: hal-02202225 \\ https://hal.science/hal-02202225}

Submitted on 31 Jul 2019

HAL is a multi-disciplinary open access archive for the deposit and dissemination of scientific research documents, whether they are published or not. The documents may come from teaching and research institutions in France or abroad, or from public or private research centers.
L'archive ouverte pluridisciplinaire HAL, est destinée au dépôt et à la diffusion de documents scientifiques de niveau recherche, publiés ou non, émanant des établissements d'enseignement et de recherche français ou étrangers, des laboratoires publics ou privés. 


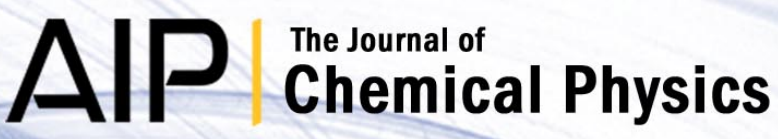

A fresh look at dense hydrogen under pressure. I. An introduction to the problem, and an index probing equalization of $\mathrm{H}-\mathrm{H}$ distances

Vanessa Labet, Paulina Gonzalez-Morelos, Roald Hoffmann, and N. W. Ashcroft

Citation: J. Chem. Phys. 136, 074501 (2012); doi: 10.1063/1.3679662

View online: http://dx.doi.org/10.1063/1.3679662

View Table of Contents: http://jcp.aip.org/resource/1/JCPSA6/v136/i7

Published by the American Institute of Physics.

Additional information on J. Chem. Phys.

Journal Homepage: http://jcp.aip.org/

Journal Information: http://jcp.aip.org/about/about_the_journal

Top downloads: http://jcp.aip.org/features/most_downloaded

Information for Authors: http://jcp.aip.org/authors

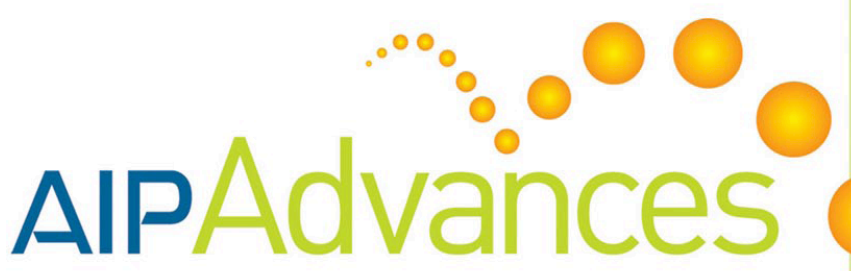

Explore AIP's new open-access journal

Article-level metrics now available

Join the conversation! Rate $\&$ comment on articles 


\title{
A fresh look at dense hydrogen under pressure. I. An introduction to the problem, and an index probing equalization of $\mathrm{H}-\mathrm{H}$ distances
}

\author{
Vanessa Labet, ${ }^{1, a)}$ Paulina Gonzalez-Morelos, ${ }^{1}$ Roald Hoffmann, ${ }^{1, b)}$ and N. W. Ashcroft ${ }^{2}$ \\ ${ }^{1}$ Department of Chemistry and Chemical Biology, Cornell University, Baker Laboratory, \\ Ithaca, New York 14853, USA \\ ${ }^{2}$ Laboratory of Atomic and Solid State Physics and Cornell Center for Materials Research, \\ Cornell University, Clark Hall, Ithaca, New York 14853, USA
}

(Received 19 September 2011; accepted 6 January 2012; published online 15 February 2012)

\begin{abstract}
In the first of a series of four papers on hydrogen under pressure, and its transitions from an initiating molecular state, we begin by defining carefully the problem, and setting the distance scale of interactions of protons and electrons in molecular aggregates of the first of the elements. Following a review of the experimental situation, in particular the phase diagram of hydrogen, in as much as it is known, and the behavior of its vibrons and rotons, we move onto the setting up of a numerical laboratory for probing the underlying physics and chemistry of interactions in hydrogen as the pressure increases. The laboratory consists of the preferred static structures emerging from calculations on the system in the range of $1 \mathrm{~atm}$ to $500 \mathrm{GPa}$, those of Pickard and Needs. The intermolecular (inter-pair) $\mathrm{H} \cdot \mathrm{H}$ separations naturally decrease with increasing pressure, first rapidly so, then more slowly. The intramolecular (intra-pair) $\mathrm{H}-\mathrm{H}$ distances vary over a much smaller scale $(0.05 \AA)$ as the pressure increases, first decreasing, then increasing, and finally decreasing. We define an equalization function to gauge the approach to equality of the first neighbor and shortest next neighbor $\mathrm{H}$ (proton) separations in this numerical laboratory. And we find that metallization is likely to occur before bond equalization. (C) 2012 American Institute of Physics. [http://dx.doi.org/10.1063/1.3679662]
\end{abstract}

\section{INTRODUCTION}

\section{A. Physical description of the problem}

Prolific in its combination with other elements in the periodic table, hydrogen is strikingly active in its chemistry. As the most abundant element in the visible universe by far, the physical states taken up by pure hydrogen as its mean density is varied are also remarkable in their diversity.

In its prominent atomic form, a single electron-mass $m_{e}$, charge $-e$, and spin $1 / 2$ - can be bound to a single proton-mass $m_{p}\left(1836 m_{e}\right)$, charge $+e$, also with spin $1 / 2$. For electron and proton coordinates $\vec{r}_{e}$ and $\vec{r}_{p}$, the Hamiltonian for this fundamental system taken as isolated is just,

$$
\hat{H}=\hat{T}_{p}+\hat{T}_{e}-\frac{e^{2}}{\left|\vec{r}_{e}-\vec{r}_{p}\right|},
$$

where $\hat{T}$ are the standard kinetic energy operators (here, the center of mass is in a state of uniform motion). Thus, for example, $\hat{T}_{p}=-\left(\hbar^{2} / 2 m_{p}\right) \vec{\nabla}_{p}^{2}$ with $\hbar$ being the reduced Planck's constant; in Eq. (1) $\left(e^{2} / r\right)=V_{c}(r)$ is the Coulomb potential energy of interaction. The general quantum description of the nature of the states of a charged particle in the presence of a point multipole is an extensive one; here an electron is in the presence of a point monopole, one of the key initiating problems of quantum mechanics.

\footnotetext{
a) Present address: LADIR (UMR 7075 CNRS/UPMC), Université Pierre et Marie Curie, 4 Place Jussieu, 75252 Paris Cedex 05, France.

b) Author to whom correspondence should be addressed. Electronic mail: rh34@cornell.edu.
}

The ground state energy of the electron-proton pair in the frame of the proton defines the atomic scale of energy (the Rydberg) via the measurable lowest total energy of the hydrogen atom,

$$
R=\frac{1}{2} m_{e} \frac{e^{4}}{\hbar^{2}}=13.59 \mathrm{eV} .
$$

It is the hydrogen problem (1) that also defines the natural atomic scale of length, the Bohr,

$$
a_{0}=\frac{\hbar^{2}}{m_{e} e^{2}}=0.529 \AA \text {, }
$$

in terms of which $R=\left(e^{2} / 2 a_{0}\right)$. In the atomic ground state, where the electron spin may take one of two projections (and likewise the proton spin), the average electron density near the proton is cusp-like and follows a distribution proportional to $\exp \left(-\left(2 r / a_{0}\right)\right)$. This immediately implies that the gradient of this density distribution at the proton is proportional to the density itself, a property that is far more general for dense assemblies of hydrogen. If a fixed point charge, here a proton, is introduced into any electronic distribution $\rho$ then it is well known (see below) that the average gradient of the ensuing distribution at the point charge is proportional to the charge density there itself. ${ }^{1,2}$

To the proton-electron system above, a second electron can be added. The ground state (of what is now $\mathrm{H}^{-}$) is bound and its existence was shown by Bethe ${ }^{3}$ but the system has no excited states that are bound, as proven by Hill. ${ }^{4}$

Likewise, consider the three-charged-particle system formed by adding a second proton to the proton-electron system above. The Hamiltonian is now $\hat{H}=\hat{T}_{e}+\hat{T}_{p, 1}+\hat{T}_{p, 2}$ 
$+\ldots$ and it describes the familiar $\mathrm{H}_{2}{ }^{+}$, the first molecule for which an exact quantum-mechanical solution was obtained. More to the point for what follows, despite the quantummechanical nature of this three-particle system, it is interesting to realize how more convenient it is for us to adopt a near classical mode of thinking for the protons, with, on account of their mass, coordinates assigned to them, and especially in the context of the emerging term "bond" which we shall be using throughout. The venerable and historic concept of a chemical bond deserves a multi-volume exposition; below we will describe briefly the various experimental and theoretical measures associated with this construct for hydrogen.

For this problem the protons are no longer in states of uniform motion; the Hamiltonian for this 3-body system is just,

$$
\begin{aligned}
\hat{H}= & \hat{T}_{e}+\hat{T}_{p, 1}+\hat{T}_{p, 2}+e^{2} \\
& \times\left[\frac{1}{\left|\vec{r}_{p, 1}-\vec{r}_{p, 2}\right|}-\frac{1}{\left|\vec{r}_{p, 1}-\vec{r}_{e}\right|}-\frac{1}{\left|\vec{r}_{p, 2}-\vec{r}_{e}\right|}\right],
\end{aligned}
$$

for which standard treatments frequently take the mass of the proton as initially infinite. The two protons and the electron they are sharing are then bound together at a proton-proton separation which is very close to $2 a_{0}$, through what we can call a 1 electron- 2 center covalent bond. The corresponding ground state energy of Eq. (2) is $-0.195 \mathrm{Ry}$ $(-2.65 \mathrm{eV})$.

Appearing here is a first characteristic length of the hydrogen problem, to be discussed in detail in what follows but in which the number of protons (and electrons) will eventually take on macroscopic values. Other emerging proton separations will also take on very different but quite density dependent measures. Assigning the proper mass to the proton in Eq. (2), leads, via a harmonic approximation, to a zeropoint energy arising from intramolecular vibrational motion of $0.0106 \mathrm{Ry}$, or $144 \mathrm{meV}$ per molecular cation, and a root mean square displacement of $0.12 \AA$ or $0.23 a_{0}$. It is evident that the two protons also establish, via their relative coordinates, a rotational problem with an associated rotational quantum number, $J$ (and associated displacements when eventually hindered in an extended system).

A second, and perhaps far more familiar proton-proton distance arises in the neutral 4-body problem constituted by two protons (coordinates $\vec{r}_{p, 1}$ and $\vec{r}_{p, 2}$ ) and two electrons (coordinates $\vec{r}_{e, 1}$ and $\vec{r}_{e, 2}$ ). Its bound states lead to the ubiquitous hydrogen molecule.

In order to link to the neutral extended state systems developed below, we may introduce the familiar Hamiltonian for this system but in the following way: index the constituents by an $\alpha$, in such a way that $\alpha=1$ corresponds to protons and $\alpha$ $=2$ to electrons. Let their corresponding sets of positional coordinates be $\left\{\vec{r}_{j, p}\right\}$ and $\left\{\vec{r}_{i, e}\right\}$, whose domain is a macroscopic volume $V$ and where $i$ and $j$ both run from 1 to $N$ in a neutral system. Then the Hamiltonian is just,

$\hat{H}=\sum_{\alpha, i}\left\{\hat{T}_{i, \alpha}+\frac{1}{2} \sum_{\left[\alpha^{\prime}, i^{\prime}\right] \neq[\alpha, i]}(-1)^{\alpha+\alpha^{\prime}} \frac{e^{2}}{\left|\vec{r}_{i, \alpha}-\vec{r}_{i^{\prime}, \alpha^{\prime}}\right|}\right\}$.

Again, for the $N=2$ problem described by Eq. (3) (the neutral hydrogen molecule) the first step is to take the proton masses as infinite. The ground state is then one with an energy of $-0.178 \mathrm{Ry}$ or $-2.42 \mathrm{eV}$ per electron or per proton, and again it leads to a highly specific proton-proton separation, now of $1.40 a_{0}$. For what follows this constitutes a second important length in the hydrogen problem, characteristic of the presence of a 2 electron- 2 center bond linking together the four particles. Its systematic and relative development for extended states of hydrogen of increasing average density is the quite major concern in what follows.

If the protons are endowed with their proper masses, proton dynamical energies associated with intramolecular vibrational motion are considerable, amounting to $\sim 0.010 \mathrm{Ry}$ or $136 \mathrm{meV}$ per proton in the ground state of this 4 particle system. As will become apparent below, these zero point energies will be seen to remain considerable for dense arrangements of hydrogen especially when viewed in terms of structural energy differences per proton. And as with $\mathrm{H}_{2}{ }^{+}$, the $\mathrm{H}_{2}$ problem once again has associated orientational physics with quantum numbers $J .^{5}$

We re-emphasize the appearance of the length $1.40 a_{0}$, as the average proton-proton separation in the $N=2$ proton (and electron) or $\mathrm{H}_{2}$ molecule problem. For it is clear that when the system is extended to macroscopic values of $N$, but in a volume $V$ that can be experimentally altered (by, for instance, application of static or dynamic pressures), then other lengths of immediate importance to both the emerging bondand band-descriptions must arise. Of crucial interest will be, for example, the average proton-proton spacings between different but neighboring pairs or molecules. As $V / N$ is progressively reduced, for example, by systematic increase of external pressure, this might well be expected to approach the average proton-proton spacing within a molecule as has been introduced above. It is then of considerable interest to ask just how an approach towards "equalization" takes place in detail, and then what role structures that minimize the enthalpies (or even Gibbs energies in a thermodynamic context) play in this progression.

It is also to be emphasized that Hamiltonian (3) as written, and for $N>2$, needs no modification for an extended neutral system of protons and electrons. It will describe all phases of hydrogen. With a doubling of the proton mass, and an ineluctable change in quantum statistics (from FermiDirac to Bose-Einstein) it will also describe all phase of deuterium. Similar remarks apply to tritium where in the course of tripling the proton mass there is a return to Fermi-Dirac statistics.

At this point it is important to emphasize that Hamiltonian (3) for macroscopic $N$ describes a formally isolated system; we are not engaged in what follows in any statistical discussion of possible distributions of the states of $\mathrm{H}$ as might emerge should our system be connected in a thermal sense to other large systems which could serve as heat baths. We will primarily be concerned with the ground states of Eq. (3) as they may evolve under the progressive changes of $V$ (mainly reductions) and for these the important functions to us for macroscopic $N$ will be the internal energy $E$ and, for comparisons between phases, the enthalpy $E+p V$, where for constant $N$ we will take the pressure $p$ to be $-(d E / d V)$, all evaluated in the ground state. We note that experimental data 
for dense hydrogen is not taken in a ground state, but is often not far removed from it.

The protonated molecular hydrogen $\mathrm{H}_{3}{ }^{+}$, known to be the most abundantly produced interstellar molecular ion, also constitutes a fascinating system, with its 3 protons bound by 2 electrons. It adopts a perfect equilateral triangular structure, with an inter-proton separation of $1.6 a_{0}$, intermediate between the characteristic $\mathrm{H}-\mathrm{H}$ bond length in $\mathrm{H}_{2}\left(1.40 a_{0}\right)$ and $\mathrm{H}_{2}{ }^{+}\left(2.0 a_{0}\right)$. It is an interesting commentary on the role of correlation that when an electron is added to $\mathrm{H}_{3}{ }^{+}$the resulting neutral system is not stable.

With respect to the issue of possible equalization of intra-pair and inter-pair proton-proton separations in solid hydrogen under pressure to be taken up below, we may note that a very high density ground state limit of Eq. (3) indicates some interesting possibilities following from the argument that the effective proton-proton interaction must eventually reflect screening by very wide-band electrons. These are distributed in states with a sharp cutoff in occupation in reciprocal space. In real space this must lead to oscillatory behavior in the screened proton-proton interactions (a manifestation of the Friedel oscillations). Since the wavelengths of these are non-commensurate with lattice constants in crystalline arrangements, this leads to an expectation that near-neighbor separations will not be commensurate either. The consequence in terms a possible departure from equalization, is, of course, subject to the effects of zero-point excursions.

\section{B. Presentation of the study}

In this series of four interconnected papers, we will take a fresh look at the evolution, in dense hydrogen, of the protonproton separations as the pressure increases, and in a particular limit, namely one where proton masses are taken as infinite (which will be referred to as the static approximation). However, in order to preserve further links with the extant literature we will constantly make reference to $\mathrm{H}-\mathrm{H}$ separations, rather than proton-proton separations (the separation between the formal high mass nuclei of the initiating hydrogen, $\mathrm{H}$, atoms). We will focus on the $\mathrm{H}-\mathrm{H}$ separation between protons involved in the same $\mathrm{H}_{2}$ unit-we will refer to this separation either as the shortest $\mathrm{H}-\mathrm{H}$ distance, the intramolecular $\mathrm{H}-\mathrm{H}$ distance, or the $\mathrm{H}-\mathrm{H}$ bond length. And we will also make good use of the shortest $\mathrm{H}-\mathrm{H}$ separation between neighboring $\mathrm{H}_{2}$ units, which we will call the second shortest $\mathrm{H}-\mathrm{H}$ separation or the shortest intermolecular $\mathrm{H}-\mathrm{H}$ separation. The chemistry and physics of the equalization of $\mathrm{H}-\mathrm{H}$ distances in hydrogen under pressure will therefore be taken up in four stages.

In the first stage, we will study the evolution of the intramolecular and shortest intermolecular $\mathrm{H}-\mathrm{H}$ distances in some candidate structures for crystalline hydrogen from $P$ $=1 \mathrm{~atm}$ to $P=500 \mathrm{GPa}$. Structures in this pressure range have been recently proposed by Pickard and Needs, ${ }^{6}$ emerging from their $a b$ initio random structure searching (AIRSS) algorithm, ${ }^{7,8}$ a procedure specifically designed to predict crystalline structures. Importantly, we will propose a new in- dex to measure the degree of equalization of the intra- and shortest intermolecular $\mathrm{H}-\mathrm{H}$ distances in these phases as the pressure increases.

In the second stage, we will propose some explanations, physical and orbital or chemical, for the particular behavior found for the intramolecular $\mathrm{H}-\mathrm{H}$ separation in hydrogen under pressure, these based on a complementary study of discrete molecular models. ${ }^{9}$

Then, in the third stage, we will return to the structures proposed by Pickard and Needs for crystalline hydrogen, and examine how the intramolecular $\mathrm{H}-\mathrm{H}$ distances in these may be understood from the arguments advanced in the second paper. ${ }^{10}$

In the fourth stage, the question of equalization of the intramolecular and shortest intermolecular $\mathrm{H}-\mathrm{H}$ distances in dense hydrogen under pressure will be studied from another perspective-by constructing several hypothetical structures having the special feature of allowing continuous transformation of a molecular phase into a monatomic one through the evolution of just a single structural parameter (and it is here we will see the golden mean appearing). ${ }^{11}$

The condensed phases of the first element would appear to present simple problems for the physics and chemistry of our time; they do not. Our four papers, taken together, will provide a fresh perspective of the chemical and physical processes at work in solid hydrogen as its density increases.

\section{A word about the terminology we will use}

\section{The chemical bond}

As we begin to think of reasons for the observed and calculated $\mathrm{H}-\mathrm{H}$ separations, it is appropriate to try to be more precise here on the meaning of some words and concepts that we are using extensively throughout this series of papers and indeed in the literature. First, when we use the word "hydrogen," we imply the element, and not whether it is diatomic $\mathrm{H}_{2}$ or monatomic $\mathrm{H}$. Second, we speak of bonds-of their "real" presence, of their partial existence as the nuclei and electrons of hydrogens come closer together under pressure, inducing a reorganization of electron density. But how do we know a bond is there? Or is not? The bond is a wonderfully useful chemical concept, yet one that is not that easy to define. Robert S. Mulliken wisely said "I believe the chemical bond is not so simple as some people seem to think. ${ }^{12}$ " As we will see, hydrogen in dense form illustrates this perfectly.

Into the definition of a bond there enter experimental measures. First and foremost among these is the average internuclear separation, obtained for example from electron, $\mathrm{x}$-ray, and neutron diffraction, or from microwave spectroscopy. The various methods of structure determination consider the molecule as a vibrating quantum object, of course. One can also measure the energies of bonds thermodynamically, and through a force-field analysis, get a measure of at least harmonic force constants determining the normal modes of vibration of a molecule. And then move on further to anharmonicities. The frequencies observed are clearly dependent on mass, which in quantum terms obviously enters into the 
very localizability of the objects being bonded. There are also magnetic consequences of binding (chemical shifts, coupling constants) and spectroscopic ones, as well.

On the theoretical side we have various ingenious analyses of the wave functions calculated from approximate solutions of Schrödinger's equation, or of the associated densities. The measures devised include bond orders and other indices, such as overlap populations; the identification of bond paths and bond critical points in Bader's quantum theory of atoms in molecules $;{ }^{13}$ the analysis can also include electron localization descriptions of several types (e.g., the electron localization function) $;{ }^{14}$ natural bond orbital analyses,${ }^{15}$ and energy decomposition schemes. ${ }^{16}$

For strong bonds, these various methods agree, for weak bonds they may disagree. Such disagreements in the end prove to be of little consequence for practical chemistry, but nevertheless provide considerable theoretical interest. One important point to realize is that there is no experimental dichotomy between the existence of a bond and the lack of a bond. The experience at normal pressure (some 700000 crystal structures determined to date, largely by $\mathrm{x}$-ray diffraction) is that for most chosen atom pairs there is observed in one or another bonding combination a range of distances, between the extremes of a full bond (or a multiple bond) and, substantially longer ranged, van der Waals or dispersion interaction minima.

\section{Molecule to metal, bonds to bands}

In the problem before us, where hydrogen makes a transition from an initially molecular system to a metal, and where bonds turn into bands, we have still another difficulty facing us. The fundamentally localized, insulator nature of hydrogen molecules separated from each other by several times their internal internuclear separation in a gas, turns, with increasing density, into a metallic system, in which former relatively free electrons may roam across the domain of what were formerly discrete molecules with localized electrons. The metallic limit is one that physicists have much experience in describing. But we are interested precisely in the in-between land, "twixt molecule and metal." What language shall we use in this intermediate land?

We have some experience in turning bonds into bands. Or to put it more precisely, we can trace out band formation from localized orbitals, and see how fairly localized orbitals can be formed from bands. In fact, in our group we have also devoted some effort to describing the detailed relationship between applying the perturbation of atomic nuclei cores to free-electron wave functions on one hand, and the wave functions that arise from linear combinations of localized atomic orbitals on the other. $^{17,18}$

So, might one nevertheless invite difficulties by taking simple notions of bonding from discrete molecules into a metallic realm, moreover into the extremes of density and pressure? Only, it seems, if one is not aware of the ambiguities and limitations of what goes into an understanding of chemical bonding at ambient conditions. With a balanced and far from dogmatic view of the chemical and physical determinants of bond formation, we may feel reasonably secure in taking the language of the $\mathrm{H}-\mathrm{H}$ chemical bond from the isolated diatomic to the metallic monatomic solid.

More problematic may be the use of the molecular terminology. As mentioned earlier, in this series we will study the evolution of the shortest and second shortest $\mathrm{H}-\mathrm{H}$ distances in some candidate structures for solid hydrogen. If there is no doubt that at low pressure $\mathrm{H}_{2}$ molecules are present and that those $\mathrm{H}-\mathrm{H}$ distances can be referred to as the intramolecular and shortest intermolecular $\mathrm{H}-\mathrm{H}$ distances, how accurate can this terminology nevertheless be as the pressure increases? In the presence of interactions, $\mathrm{H}_{2}$ molecules are perturbing each other. And in the same way that it is difficult to define an atom or a molecular fragment in a molecule, it becomes difficult to define a molecule in a dense molecular solid. Nonetheless, as we will see in Sec. II, there is experimental evidence for the preservation of the integrity of $\mathrm{H}_{2}$ pairs, or molecular pairs, until remarkably high pressures. Thus, for convenience (but fully aware of the limitations), we will use the molecular terminology, talking about intramolecular and intermolecular $\mathrm{H}-\mathrm{H}$ distances, as long as in the structures studied, each proton has one easily distinguishable closest neighbor, in a fully dynamical context.

\section{PHASES OF DENSE HYDROGEN}

How does crystalline hydrogen progress from an insulating dynamic molecular solid to a presumed metallic monatomic one? This question has been with us since 1935, when Wigner and Huntington ${ }^{19}$ first broached the possibility of a metallic modification of hydrogen under pressure. They expected, and we do so today, that at a sufficiently high pressure (high enough to induce a ninefold volume compression, at least), hydrogen will metallize. But, as the pressure increases continuously, will molecular hydrogen reach a hypothetical monatomic state via a progressive evolution of its intramolecular and shortest intermolecular $\mathrm{H}-\mathrm{H}$ distances? Or will it do so abruptly, through phase transitions accompanied by a sharp scission of dynamic and paired $\mathrm{H}_{2}$ molecules?

The metallic state of the simplest element has crystallized the attention of chemists, physicists, planetary scientists and the materials community. First we emphasize that we are not addressing in this study metallization per se, as interesting as it is. Hydrogen has definitely been metallized in ingenious and classic shock-wave experiments, ${ }^{20}$ yet the approach to it by steady compression at low temperatures still appears to be out of reach experimentally, even at pressures as high as $\sim 350 \mathrm{GPa}^{21-24}$ It is important to mention here a recent experiment by Eremets and Troyan in which the metallization of hydrogen is reported at a pressure of around 260-270 GPa and at around $295 \mathrm{~K}$, but persisting down to $\sim 30 \mathrm{~K} .{ }^{25}$ The work has aroused great interest, and some controversy. It is probably fair to say that as careful as this work is, it awaits confirmation by other experimentalists. In density functional theory (DFT) calculations metallization occurs at pressures $\sim 250 \mathrm{GPa}$ or above (see Table I); but the experience with the functionals utilized is that they underestimate bandgaps, and so would imply metallization at a pressure lower than that observed. 
TABLE I. Structures found to be the most stable by Pickard and Needs ${ }^{6}$ for solid hydrogen in its ground state up to $500 \mathrm{GPa}$, in the static latticeapproximation

\begin{tabular}{llc}
\hline \hline Pressure range & $\begin{array}{c}\text { Symmetry of the most } \\
\text { stable structure }\end{array}$ & $\begin{array}{c}\text { Is it metallic } \\
\text { (according to DFT)? }\end{array}$ \\
\hline$<105 \mathrm{GPa}$ & $P 6_{3} / m\left(Z=8^{\mathrm{a}}\right)$ & No \\
$105-270^{\mathrm{b}} \mathrm{GPa}$ & $C 2 / c\left(Z=12^{\mathrm{a}}\right)$ & No \\
$270-385 \mathrm{GPa}$ & $C m c a-12\left(Z=12^{\mathrm{a}}\right)$ & Yes \\
$385-490 \mathrm{GPa}$ & $C m c a\left(Z=4^{\mathrm{a}}\right)$ & Yes \\
$>490 \mathrm{GPa}$ & $I 4_{1} /$ amd $\left(Z=4^{\mathrm{a}}\right)$ & Yes \\
\hline \hline
\end{tabular}

${ }^{a}$ For the $\mathrm{P}_{3} / m, C 2 / c, \mathrm{Cmca}-12$, and Cmca structures, $\mathrm{Z}$ is the number of $\mathrm{H}_{2}$ molecules in the unit cell; for the $I 4_{1} / a m d$ structure, $Z$ is the number of $\mathrm{H}$ atoms in the unit cell.

${ }^{\mathrm{b}}$ When the zero-point energies are taken into account, the $C 2 / c$ structure stability region is shifted from $<75 \mathrm{GPa}$ to $240 \mathrm{GPa}$.

Let us review now the structural and spectroscopic experimental situation for hydrogen under pressure.

\section{A. Experimental data}

At very high pressures, some of the most informative experimental probes of the structure of solid hydrogen have proven to be its spectra or optically induced excitations, revealing its vibrons, phonons, rotons, and librons, linked to the translational and orientational excitations of the protons. Spectroscopic studies by infrared (IR) and Raman methods have led to the partial characterization of different phases for solid hydrogen at low temperatures. ${ }^{26}$ Crystallography, utilizing both x-ray and neutron diffraction, has also played an important role though obviously $x$-ray scattering from the electrons of $\mathrm{H}$ is exceedingly weak. Summarizing to date one finds:

- at 1 atmosphere and low temperatures, a molecular solid composed of freely rotating $\mathrm{H}_{2}$ molecules, their centers arranged in an hexagonal-close-packed ( $h c p$ ) structure, ${ }^{27}$ giving rise to a nearly spherical charge time average distribution, and characterized by intramolecular $\mathrm{H}-\mathrm{H}$ distances of $0.74 \AA\left(1.40 a_{0}\right)$, a shortest center-to-center intermolecular distances of $3.8 \AA\left(7.2 a_{0}\right)$, and a large direct band gap of $15.6 \mathrm{eV},{ }^{28}$

- and at very high pressures and quite high temperatures (as in the shock studies), a metallic phase, observed experimentally, but of unknown structure, presumably monatomic and possibly liquid (see Paper IV). At low temperatures but continuing with elevated pressures, this phase was predicted in 1968 to be a high-temperature superconductor, ${ }^{29}$ and later perhaps a superfluid. ${ }^{30}$

Between those two extremes, different phase transitions have been identified. Several phase diagrams for $\mathrm{H}_{2}$, emphasizing different $\mathrm{P}$ and $\mathrm{T}$ regimes, can be found in the literature. ${ }^{26,31-33}$ The phase diagram we show here (Figure 1) is a recent one from Hemley. ${ }^{33}$

The main points of the hydrogen phase diagram are these:

- Phase I, also known as the hexagonal-close-packed (HCP) phase, is characterized (as noted above) by freely rotating $\mathrm{H}_{2}$ molecules whose centers of mass

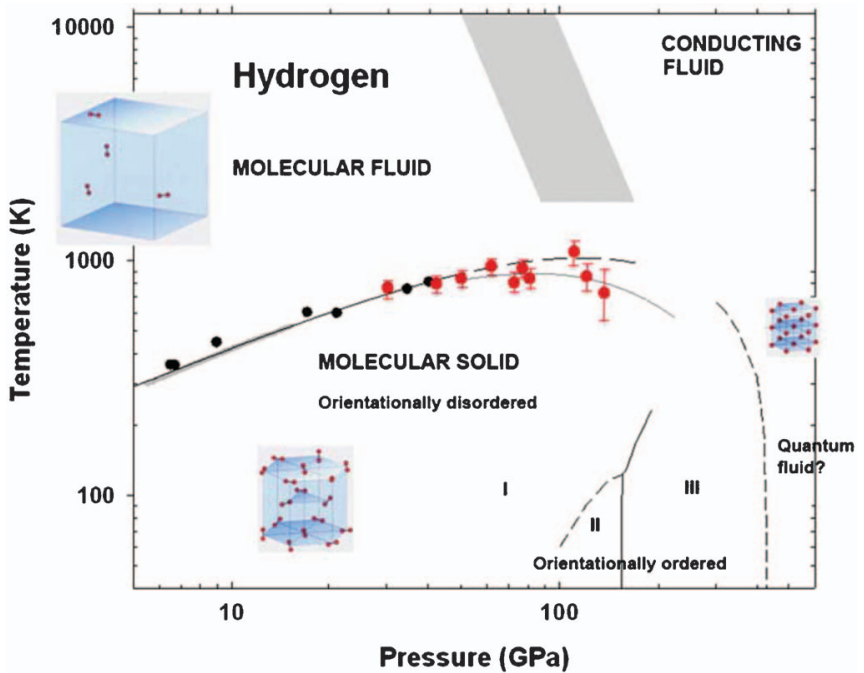

FIG. 1. Schematic phase diagram of hydrogen reproduced with permission from Hemley. ${ }^{33}$ The phase boundaries for the solid-solid Phases I, II, and III are from Mao and Hemley. ${ }^{26}$ For melting, selected experimental results are taken from Datchi et $a l .{ }^{34}$ (thick line), Gregoryanz et $a l .^{35}$ (black circles), and Subramanian et al. $^{36}$ (red circles) (for more experimental measurements, see also Refs. 37 and 38). The upper dashed line is the extrapolation from Ref. 35 and the lower grey line is the theoretical prediction of Bonev et al. ${ }^{39}$ The boundaries for the quantum fluid transition as well as transition from the molecular (insulating) to the conducting fluid are uncertain. Reprinted with permission from R. J. Hemley, High Press. Res. 30, 581 (2010). Copyright 2010, Taylor \& Francis.

are arranged in an hcp structure. ${ }^{40}$ In normal $\mathrm{H}_{2}$, at $85 \mathrm{~K}$, it persists from 1 atm to $110 \mathrm{GPa}^{41}$ a pressure where the density has been increased by a factor of 9 with respect to its value at $1 \mathrm{~atm}$ and low temperatures. In this quantum rotational solid, hydrogen remains an insulator. The Raman vibron frequency (the stretching mode at $4161 \mathrm{~cm}^{-1}$ in a gas phase $\mathrm{H}_{2}$ molecule) increases slightly from 1 atm to $30 \mathrm{GPa}$ in Phase I, suggesting an increasing repulsion of $\mathrm{H}_{2}$ molecules. The vibron frequency then decreases. The latter trend has been interpreted as a weakening of the $\mathrm{H}-\mathrm{H}$ covalent bond. ${ }^{42,43}$ Consistently, an analysis of the roton bands at low temperatures $(T=6 \mathrm{~K})$ revealed that the intramolecular $\mathrm{H}-\mathrm{H}$ distance decreases from 1 atm to $30 \mathrm{GPa}$ and then increases. ${ }^{44-46}$ This increase has been proposed to be a consequence of electron transfer from the covalent bonds to the intermolecular regions.

- A second phase, Phase II, also known as the brokensymmetry phase, ${ }^{44}$ appears at about $110 \mathrm{GPa}$ (in normal $\mathrm{H}_{2}$ at $\left.T=85 \mathrm{~K}\right)^{41}$ and is characterized by changes in the low-frequency region of the Raman and IR spectra. These changes originate with a "quantum" orientational ordering of the $\mathrm{H}_{2}$ molecules (ordering of their angular momenta), but there is no longer essentially free rotation. The motion is better described as wide-angle libration.

- At $150 \mathrm{GPa}$, a large low-temperature discontinuity in the frequency of the Raman and a strong rise in the IR molecular vibrons mark the appearance of Phase III, ${ }^{47}$ also known as the hydrogen- $A(\mathrm{H}-A)$ phase. ${ }^{48}$ To date the structure of this phase is not known definitively but 
recent X-ray powder diffraction experiments suggest that Phase III may take up a hexagonal structure. ${ }^{49} \mathrm{~A}$ new IR vibron in this phase appears at lower frequency than that of Phase II, indicating a further softening of the intramolecular vibration. And its frequency continues to decrease with increasing pressure. Moreover, the intensity of the IR vibron grows notably with pressure. ${ }^{50}$ With respect to Phase II, Phase III involves a "classical" orientational ordering of the molecules themselves. ${ }^{51}$ Complete rotation ceases but wide angle libration, periodic but not harmonic, persists. Phase III appears to be stable up to $316 \mathrm{GPa},{ }^{52}$ a pressure above which solid hydrogen has not been well-characterized experimentally.

Because of the persistence of the vibron signature of $\mathrm{H}_{2}$ molecules at such high pressures, even as a metallic state for solid hydrogen, the idea of a metallic molecular state, which has been present in the community for more than 40 years, is becoming more and more plausible. ${ }^{29,52-57}$

In the liquid phase, the experimental metallization of hydrogen by Nellis and co-workers ${ }^{20}$ is a classic experiment. Questions still remain-Is there a first-order liquid-liquid transition in the process? The work by Nellis and co-workers ${ }^{20}$ suggests metallization through a continuous process, while studies by Fortov et al..$^{58}$ argue for the presence of a plasma phase transition. Very recently, a first-order liquid-liquid phase transition has been suggested in simulation studies by Morales et al. ${ }^{31}$

\section{B. Theoretical studies}

\section{Quantum mechanical statement of the problem}

As outlined above (see Eq. (3)) a neutral assembly of $N$ protons and $N$ electrons is confined in a macroscopic volume $V$; the familiar linear measure of inverse density $(V / N)$ is the dimensionless Wigner-Seitz radius $r_{s}$, and is defined as

$$
\frac{4}{3} \pi r_{s}^{3} a_{0}^{3}=\frac{V}{N}
$$

where $a_{0}$ is the Bohr radius, introduced, as noted above, by the problem of atomic hydrogen.

In approximate terms, and in Bohrs, the average nearneighbor $\mathrm{H}-\mathrm{H}$ separation must be $2 r_{s}$. From the measured low temperature density of hydrogen, at one atmosphere, the value of $r_{s}$ is 3.12, and this must emerge from a minimization of the enthalpy of the Hamiltonian (Eq. (3)) introduced above. A crucial consequence of the strict Coulomb forms of all interactions is a rigorous scaling property of the ground state energy of Eq. (3). Quite independent of any eventual structure for the proton system, the energy per electron (or per proton) can be shown from the virial and Hellmann-Feynman theorems to be a function of $m_{e} r_{s}$ and of $m_{p} r_{s}$, and in fact takes the form

$$
\frac{E}{N}=\frac{f\left(m_{e} r_{s} ; m_{p} r_{s}\right)}{r_{s}}
$$

within the standard Born-Oppenheimer approximation. ${ }^{59}$ Use of the virial theorem, in particular, leads to
$\langle\hat{T}\rangle=-\left(d / d r_{s}\right)\left(E r_{s}\right)$ for the kinetic energy, and $\langle\hat{V}\rangle=\left(1 / r_{s}\right)\left(d / d r_{s}\right)\left(E r_{s}^{2}\right)$ for the Coulomb interaction energy. All three results provide exact constraints on any numerical or simulation procedure used to determine the ground state energy (and later the enthalpy) for the hydrogen problem.

The treatments of Eq. (3) via modern electronic structure methods almost invariably resort to infinitely massive protons. Given that the vibron in the neutral hydrogen molecule has an energy of $\sim 500 \mathrm{meV}$ and that the rotational equivalent (say for a $J=0$ to $J=2$ transition) is $\sim 44 \mathrm{meV}$, structural transitions for infinitely massive protons involving energy differences per proton much less than this (and as will be seen this is almost the norm) will eventually require treatments that deal with dynamics on a self-consistent basis, at the least.

It is clear that for a neutral system of point charges their microscopic densities cannot be homogeneous. For fixed positions of the protons situated on a lattice, the electronic density will be periodically inhomogeneous, and within the BornOppenheimer separation of time scales the electron density at each proton is also constrained by the rigorous cusp theorem of Kato. ${ }^{1,2}$ If the electronic density is taken to be $\rho_{e}(\vec{r})$, then the first theorem of Hohenberg and Kohn further demands that the ground state energy of the electron system is a unique functional of $\rho_{e}(\vec{r})$, and this provides the pathway to determination of a structure which for a given VIN (experimentally fixed by choice of pressure) will yield the lowest enthalpy. Later this has to be extended to include the energies associated with proton dynamics in what is a strongly coupled system and also of course to a fully thermodynamic description including entropic contributions.

What follows is to be viewed within the limitations explicitly listed above (and to be spelled out in a section that follows) - we will follow the trend towards equalization of $\mathrm{H}-\mathrm{H}$ separations in dense hydrogen but as is revealed in static calculations. From these static calculations, we will attempt to glean the underlying physical and chemical principles shaping the bonding and structure of the system and also comment on the possible role of proton dynamics.

\section{A numerical laboratory}

In parallel with the experimental characterizations of the different phases of solid hydrogen at low temperatures and high-pressures, considerable theoretical effort has been expended in attempting to determine the associated structures. If in the earlier days useful results have been obtained from perturbation theory and pair potential approximations, currently three main approaches are now prominently used: Density functional theory simulations for static lattices, eventually including zero-point motion effects a posteriori, ${ }^{6,57,60-63} a b$ initio molecular dynamics ${ }^{64,65}$ - the DFT-based path-integral molecular dynamics method of Kitamura et al. ${ }^{65}$ is currently one of the most highly developed approaches to take into account proton quantum effects - and full quantum Monte Carlo methods, with particular mention of the pioneering work of Ceperley and co-workers. ${ }^{6-68}$ All of these approaches, extensive and thorough as they are, are subject to the limitations, 


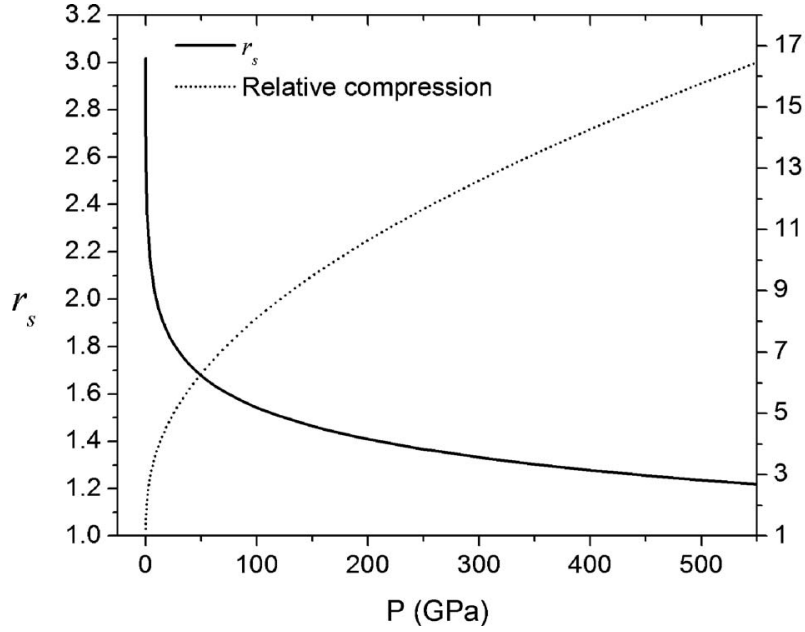

FIG. 2. Relation between pressure $P$, the $r_{s}$ parameter $\left[(4 \pi / 3)\left(r_{s} a_{0}\right)^{3}\right.$ $=V / N]$ and relative compression in solid hydrogen in its ground state. Notice the rapidity, so far as $r_{s}$ decrease goes, with which hydrogen is initially compressed.

which we voiced explicitly above, and will rehearse below. Taken together, the existing calculations can be viewed as a set of numerical experiments on dense hydrogen that carries with it quantitative information of very significant value. Our aim in this paper is to use a subset or selection of this considerable experience to garner an understanding of the physical and chemical factors underlying the transition of hydrogen from an insulating molecular solid, to a conducting phase in which proton pairing or $\mathrm{H}_{2}$ molecules (certainly highly perturbed from their low density state) can persist, or in which one can see only protons themselves, even quite mobile, and delocalized electrons.

A specific numerical subset or "laboratory" we have chosen to help us formulate ideas on the molecular to monatomic solid transition is the comprehensive study by Pickard and Needs, ${ }^{6}$ on the phase diagram of hydrogen in its ground state, and for pressures up to $P=500 \mathrm{GPa}$, using AIRSS. ${ }^{7,8}$ This paper is one of the more detailed quantum mechanical studies of hydrogen in range $P \leq 500 \mathrm{GPa}$; it takes hydrogen from $r_{s}=3.12$ to $r_{s}=1.23$ (see Eq. (4) for definition of $r_{s}$, and Figure 2 for the correspondence between pressure $P$, relative compression and the $r_{s}$ parameter in that pressure regime). Note that the AIRSS methodology and another structural prediction method based on particle swarm optimization have recently been used to examine the ground state structures of atomic metallic hydrogen at even higher pressures (500 GPa $<P<5 \mathrm{TPa}$ corresponding to $r_{s} \sim 1.23$ to $\left.r_{s} \sim 0.86\right){ }^{69,70}$ The symmetry of the structures found to be the most stable in Ref. 6 is indicated in Table I, with the pressure range of stability associated. As we will see, structures $P 6_{3} / \mathrm{m}, \mathrm{C} 2 / \mathrm{c}, \mathrm{Cmca}$ 12 and $\mathrm{Cmca}$ involve $\mathrm{H}_{2}$ molecules, while the high-pressure $I_{1} /$ amd structure corresponds to a monatomic phase. It is important to note that the static calculations reported here cannot describe a rotational or librational solid.

As mentioned above, invoking the presence of molecules in an extended structure is not unambiguous, especially under pressure, when, within our chosen terminology, intermolecular distances become comparable to intramolecular distances.

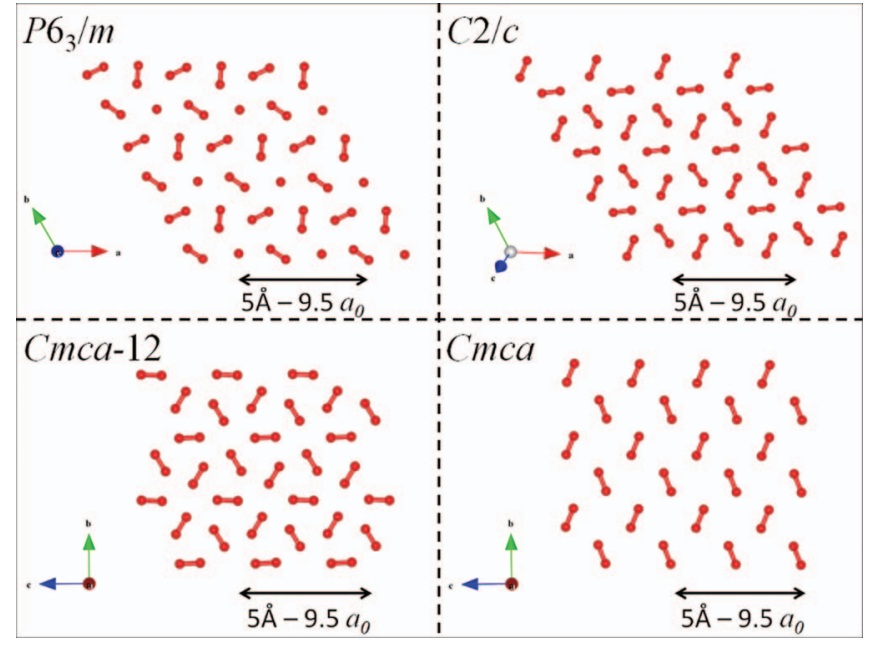

FIG. 3. A layer of the $P 6_{3} / m, C 2 / c, \mathrm{Cmca}-12$, and $\mathrm{Cmca}$ structures at $P=300 \mathrm{GPa}\left(r_{s}=1.33\right.$-relative compression of 12.6). In the $P 6_{3} / \mathrm{m}, \mathrm{Cmca}$ 12, and Cmca structures the layers are arranged in an ABA fashion; in the $C 2 / c$ structure they are arranged in an ABCDA fashion.

We will come back to this later, once we will have studied the evolution of the $\mathrm{H}-\mathrm{H}$ distances in the structures. But for now, by stating that $P 6_{3} / m, C 2 / c, C m c a-12$, and Cmca structures involve $\mathrm{H}_{2}$ molecules, we simply imply that in those structures each proton has but one closest neighbor.

a. Equation of state. Before going further, it can be helpful to have in mind an idea of the relative compression induced by a given external pressure, generally at low temperatures. Figure 2 shows the relation between pressure, relative compression and the Wigner-Seitz $r_{s}$ parameter in solid hydrogen in its ground state, and as it emerges from the equation of state proposed very recently by Caillabet et al. for the molecular hydrogen solid. ${ }^{71,72}$

b. Four molecular phases of hydrogen. The four molecular phases of Table I $\left(\mathrm{PG}_{3} / m, C 2 / c, C m c a-12\right.$, and $\left.\mathrm{Cmca}\right)$ can be described as layered, a layer of each being shown in Figure 3. Structure $I 4_{1}$ /amd is shown in Figure 4. Additional views of these five structures can be found in the supplementary material (supplementary material, Figures S1-S5 (Ref. 73)).

Structure $P 6_{3} / \mathrm{m}$, which is the most stable arrangement in the ground state in the pressure range corresponding to Phases I and II, is distinguishable from the three others molecular

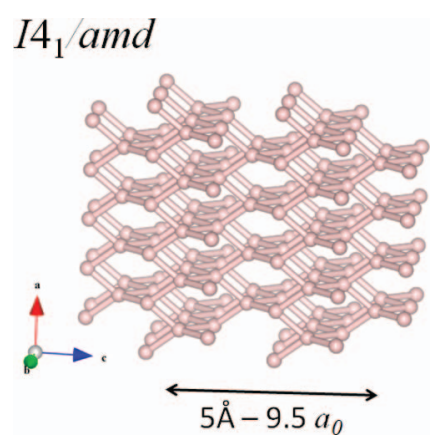

FIG. 4. View of the $I 4_{1} /$ amd structure at $P=500 \mathrm{GPa}\left(r_{s}=1.23\right.$-relative compression of 15.8). 
phases by the fact that one $\mathrm{H}_{2}$ molecule out of four has its bond lying not in the layer plane but perpendicular to it. All in all, four different orientations of the $\mathrm{H}_{2}$ molecules are therefore involved. Phase I, as we mentioned, is a fully rotational state, so the assignment of a definite $\mathrm{H}_{2}$ orientation is to some extent misleading. It is not possible to reproduce a rotational solid in a static approximation. That one indeed has a rotational solid in structure $P 6_{3} / m$ in the pressure range of Phase I is supported by calculations we have carried out, showing that the barrier to rotate any single $\mathrm{H}_{2}$ molecule in this structure is less than $1 \mathrm{meV} /$ proton (at $1 \mathrm{~atm}$ ) (recall that the zeropoint energy at $1 \mathrm{~atm}$ is $\sim 125 \mathrm{meV} /$ proton). There are other candidate structures for Phase II in the literature, such as the $P 2_{1} / c,{ }^{57} P c a 2_{1},{ }^{64}$ and $P 2_{1} / c-24$ (Ref. 74) structures. All of them, as many others, are very close in energy (a few meV per proton) and we strongly believe the arguments developed in this paper as well as in the three others of the series are little dependent on structure in the low-pressure regime; this is why we decided to keep with the $P \sigma_{3} / m$ structure as the representative for Phase II.

In the $C 2 / c$ structure-proposed as a competing structure for Phase $\mathrm{III}^{6,75}$-and in the Cmca-12 structure, which is characterized by a similar arrangement of the $\mathrm{H}_{2}$ molecules into triangles, the number of different directions for the $\mathrm{H}_{2}$ molecules, is reduced to three, and then further to two in the Cmca structure. In the monatomic I4 1 /amd structure, each hydrogen atom is 4-coordinated, and is involved in 6-membered rings.

An important aspect of the enthalpics of these structures is how close to each other they are throughout this entire and very substantial pressure range. No more than $10 \mathrm{meV} /$ proton separates all four molecular structures up to $500 \mathrm{GPa}$. One can see immediately why one might therefore think that $\mathrm{H}_{2}$ under pressure may well prefer to adopt a liquid phase. And yet, quite distinct physical phenomena ensue, at definite pressures - the rapid turning on of the intensity of the IR active vibron in Phase III being one such.

c. A word about the static view of a dynamical system. Before going further, let us take some time here to review the meaning and implications of the structures shown in Figures 3 and 4. In their study and as noted, Pickard and Needs searched for structures within the framework of the static approximation as defined earlier, i.e., assuming an infinite mass for the protons. Figures 3 and 4 can be interpreted as the complete representations of those static structures. In reality, even in the ground state, $\mathrm{H}_{2}$ molecules as quantum oscillators, are vibrating $\left(\sim 10^{15}\right.$ vibrations per second for the $\mathrm{H}-\mathrm{H}$ bond stretching) and in Phase I rotating $\left(\sim 10^{13}\right.$ rotations per second for the $\mathrm{H}_{2}$ molecule rotation). This is irreducible zero-point motion, and the associated energy is quite large because of the light mass of the protons (or deuterons). These realities bring a certain but necessary caution to attributing any great realism to the static pictures shown in Figures 3 and 4.

Though the static approximation may be a reasonable starting point for the description of the solid state of hydrogen, it is obviously unphysical for the liquid state. There are some strong indications of the possibility of a liquid state for hydrogen even in its ground state, in a range of pressures at elevated pressures. ${ }^{30}$ Thus, it seems important to en- sure that the static approximation holds in the pressure range considered in the study. Lindemann's empirical law for melting provides an easy way to evaluate, at least approximately, the pressure at which molecular hydrogen would melt—when in classical terms the root mean vibration (RMS) amplitude $\sqrt{\left\langle u^{2}\right\rangle}$ of $\mathrm{H}_{2}$ oscillators reaches about $12 \%$ of the shortest $\mathrm{H}_{2}-\mathrm{H}_{2}$ separation. In fact, for quantum or ground state melting, it has been shown that the RMS amplitude should reach $30 \%$ of the separation. ${ }^{76}$ (The Lindemann parameter in helium is $\sim 28 \%$.) For metallic hydrogen, it is predicted to be $\sim\left(m_{e} / m_{p}\right)^{1 / 4}(\sim 15 \%)$ in the Bohm-Staver approximation for sound speeds ${ }^{77}$ and its subsequent application in the Debye model. In the non-interacting harmonic quantum oscillator approximation, the RMS amplitude of the $\mathrm{H}-\mathrm{H}$ bond stretching vibrations due to the zero-point motion is given by formula (5),

$$
\sqrt{\left\langle u^{2}\right\rangle}=\sqrt{\frac{\hbar}{2 \mu \omega_{0}}},
$$

with $\mu$ the reduced mass of the oscillator and $\omega_{0}$ the angular frequency of the normal mode of vibration.

By using the $\omega_{0}$ value of the free $\mathrm{H}_{2}$ molecule stretching vibration, one gets $\sqrt{\left\langle u^{2}\right\rangle} \approx 0.09 \AA \approx 0.17 a_{0}$. Thus in solid molecular hydrogen these critical displacements are reached when the shortest intermolecular distance is about $0.3 \AA, \sim 0.57 a_{0}$ which is actually smaller than the $\mathrm{H}-\mathrm{H}$ bond length of a free $\mathrm{H}_{2}$ molecule. This indicates that the translational zero-point motion may not of itself induce melting of a molecular phase. The orientational degrees of freedom, an order of magnitude softer, are far more likely to be the source of such a transition as well as the source of purely orientational restructuring. However, considering $\mathrm{H}_{2}$ molecules as 3D harmonic rotors, the RMS amplitude is about 0.24 A, i.e., 0.46 $a_{0}$ and this represents $30 \%$ of an intermolecular distance. This estimate is not expected to be very precise, but it serves to caution us that in the molecular solid when the intramolecular $\mathrm{H}-\mathrm{H}$ separation and the shortest intermolecular separation become comparable the solid is not only compressed but may be close to its melting point even though this may well be preceded, as noted, by average orientational re-orderings reflecting combined translational and rotational displacements.

The melting of systems whose nominal Debye temperatures are in excess of their melting temperatures has been discussed by Glyde. ${ }^{78}$

We again need to be explicit about the extensive functions with dimensions of energy we use in the discussion of the geometrical preferences of the various phases. The calculations as such are carried out for ground state comparisons of the various structures and, save for the third law could be taken as corresponding to $T \rightarrow 0 \mathrm{~K}$, at which point the Gibbs energy of any system would be formally equal to its enthalpy. It is in this sense, and under the constraint of $T \rightarrow 0 \mathrm{~K}$, that we proceed to use in the remainder of this and the other papers in this series the words "enthalpy" and "enthalpic" in differentiating between structures. 


\section{PRESENTATION OF THE STUDY AND COMPUTATIONAL DETAILS}

Let us assume for now that the Pickard and Needs static structures are at least a reasonable starting point with which to formulate an approximation to the evolution of hydrogen, from $P=1 \mathrm{~atm}$ to $P=500 \mathrm{GPa}$ (i.e., from $r_{s}=3.12$ to $r_{s}$ $=1.23) .{ }^{79}$ Analyzing those five phases from a structural point of view can then help us understand how molecular hydrogen transforms into monatomic hydrogen, if it does so. For that purpose, we studied for each phase the evolution of the intramolecular and shortest intermolecular $\mathrm{H}-\mathrm{H}$ distances, considering each structure in the pressure range within which is the most stable.

Starting from the coordinates provided by Pickard and Needs (in the supplementary material of Ref. 6) we optimized the ground state static structures for different pressures between $1 \mathrm{~atm}$ and $500 \mathrm{GPa}$, using the VASP plane wave code ${ }^{80-82}$ the Perdew-Burke-Ernzerhof (PBE) generalized gradient approximation density functional, ${ }^{83,84}$ and the projector augmented-wave method ${ }^{85,86}$ with a pseudopotential cutoff radius of $0.8 a_{0}$ and a cutoff of $2000 \mathrm{eV}$ for the kinetic energy of the plane waves.

It is immediately clear that the cusp theorem cannot be satisfied for such a pseudopotential. Nonetheless, in their recent theoretical study of atomic hydrogen between $P$ $=500 \mathrm{GPa}$ and $P=5 \mathrm{GPa},{ }^{69}$ McMahon and Ceperley specifically studied the validity of the pseudopotential approximation by comparing the energies and electron densities of several structures computed with two choices of pseudopotential cutoff radius: $0.5 a_{0}$ and $0.125 a_{0}$. They concluded that a cutoff radius of $0.5 a_{0}$ was a reasonable approximation. Since we are studying hydrogen at lower pressures, we assume that our choice of a pseudopotential cutoff radius of $0.8 a_{0}$-the smallest available in VASP-is reasonable.

In each case, the cell shape and volume of the unit cells were allowed to change and the ions to relax, leading eventually to very small deviation with respect to the group symmetry used to label the structures. The k-point sets for the Brillouin-zone sampling were generated via the MonkhorstPack scheme ${ }^{87}$ and a different set was used for each structure at each pressure, in order to have in each case a grid of spacing of $2 \pi \times 0.02 \AA^{-1}\left(\sim 2 \pi \times 0.01 a_{0}^{-1}\right)$. Since we are more interested by the evolution of intramolecular and intermolecular $\mathrm{H}-\mathrm{H}$ distances in those structures than in their relative enthalpies, we did not consider the zero-point motion at this stage (though it is clearly important as argued earlier). Within this static approximation, good agreement was found with the Pickard and Needs results for the range of pressure in which each structure was stable. ${ }^{88}$

But we must emphasize again the limitations of the underlying calculations: the quantum-mechanical effects for the protons have not been taken into account in any of the computations presented in this article. As our discussion of average atomic excursions in the ground state (Sec. II) has shown, this does not mean that we think the quantum-mechanical dynamic effects can be neglected. Moreover, as already indicated, the use of a pseudopotential for the effective electron-proton interaction can induce errors in the electronic distribution as high-pressures are considered. Nor do we pretend that the present results are highly reliable from a quantitative perspective. But we do believe that they are sufficient at this stage to help us in building our chemical and physical intuition on the emerging qualitative behavior of dense hydrogen and its structural development under pressure, and then the emerging problem of equalization.

\section{ANALYSIS OF THE PICKARD AND NEEDS STRUCTURES}

The general manner in which condensed molecular systems respond to compression is reasonably well understood. ${ }^{89}$ In particular, as a first response, van der Waals or dispersion interaction-space is squeezed out. Then, at greater pressures, elements or the elements in combination try to respond to the rising density by electronic reorganization, which in many cases leads to higher coordination. And at higher pressures still, covalent bonds contract. But they can also stretch. We will discuss the latter ambiguity below.

\section{A. When the intermolecular distances decrease}

Because a $\mathrm{H}_{2}$ molecule is a three-dimensional (3D)object and because some of the molecular structures considered involve non-equivalent $\mathrm{H}_{2}$ molecules in the primitive unit cell, there are quite different ways to define the shortest intermolecular $\mathrm{H}-\mathrm{H}$ separation in the structures. We chose the following: In each structure, in the pressure range considered, all protons are involved in $\mathrm{H}_{2}$ molecule, i.e., have one close proton less than $0.8 \AA$ away from the reference proton (blue arrow). The first neighbor of each proton is thus the proton to which it is "bonded" to form an $\mathrm{H}_{2}$ unit. And the second closest neighbor(s) is (are) a proton of another $\mathrm{H}_{2}$ molecule. It is the second closest neighbor distance we focus on, highlighted by the green dashed arrow on Figure 5 . We computed the average separations of this (these) second shortest

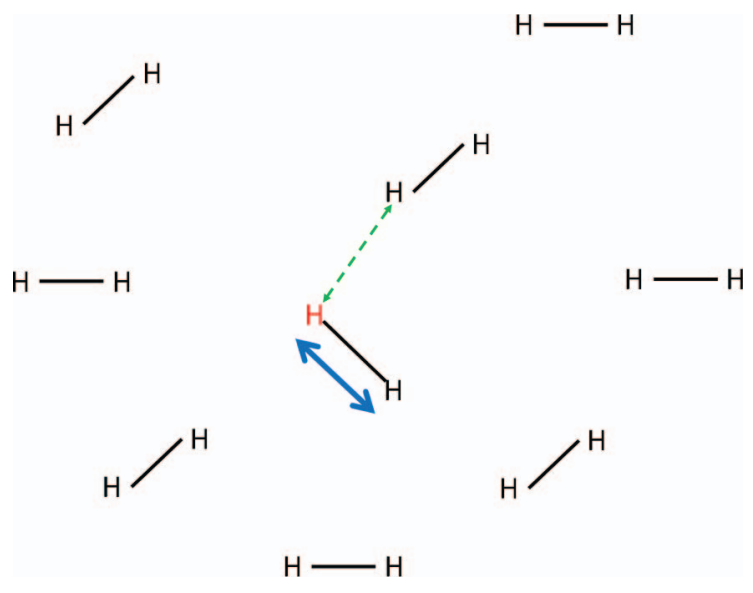

FIG. 5. Schematic representation of the definitions of the shortest intermolecular distance and intramolecular distance used in this work. The green dashed arrow and the plain blue arrow indicate, respectively, the shortest intermolecular distance between the proton, in red, and any of the other protons in black and the intramolecular distance in which the red proton is involved. Here the protons are assumed fixed. 


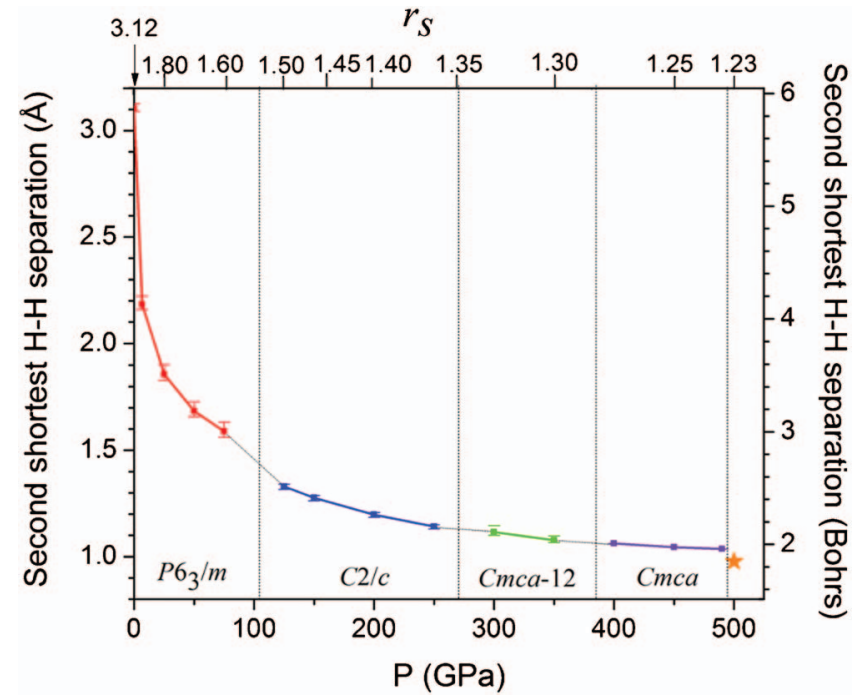

FIG. 6. Evolution of the average second-shortest $\mathrm{H}-\mathrm{H}$ distance (the shortest intermolecular $\mathrm{H}-\mathrm{H}$ separation) in various hydrogen phases. Where there are several symmetry-distinct $\mathrm{H}_{2}$ molecules in a structure, the maximal and minimal values are indicated by "spread bars." The orange star indicates the value of the shortest $\mathrm{H}-\mathrm{H}$ distance in the monatomic $I 4_{1} /$ amd structure at 500 GPa. Note the similarity to the evolution of the Wigner-Seitz radius $r_{s}$ vs $P$ shown in Figure 2.

neighbor(s) for each proton in the unit cell and then defined the shortest intermolecular $\mathrm{H}-\mathrm{H}$ distance as the value of the second shortest $\mathrm{H}-\mathrm{H}$ separation averaged over the whole unit cell.

From the perspective of the considerations given just above, the average distance between two molecules is expected to decrease when pressure increases, first dramatically (as expected from Figure 2) and then more progressively. In Figure 6 is plotted the evolution of the average shortest intermolecular $\mathrm{H}-\mathrm{H}$ separation in hydrogen under pressure in the $\mathrm{PG}_{3} / m, \mathrm{C}_{2} / c, \mathrm{Cmca}-12$, and $\mathrm{Cmca}$ molecular structures, considering each of them in the pressure range where it constitutes the most stable structure (see Table I). As a calibration, the shortest $\mathrm{H}-\mathrm{H}$ separation in the $I 4_{1} /$ amd monatomic structure at $P=500 \mathrm{GPa}$ is also indicated (orange star). The evolution of the interlayer distance with pressure can be found in the supplementary material to this paper, Figure S6. ${ }^{73}$

It must be noted that below $110 \mathrm{GPa}$ this definition of the shortest intermolecular $\mathrm{H}-\mathrm{H}$ separation may to some extent be invalid, since Phases I and II are certainly composed of freely rotating or wide-angle librating molecules, respectively. But since we are most interested in the behavior of hydrogen in Phase III, to come, where the $\mathrm{H}_{2}$ molecules are more constrained, we adopt this definition over the entire pressure range, from 1 atm to $490 \mathrm{GPa}$.

As the pressure increases from 1 atm, Figure 6 shows that the shortest intermolecular $\mathrm{H}-\mathrm{H}$ separation first falls very rapidly in the $P 6_{3} / m$ structure. In this region, $\mathrm{H}_{2}$ molecules are brought into the repulsive region of a van der Waals interaction; the energetic cost is (relatively) not too great. Colloquially one can speak (as above) of van der Waals space being squeezed out; to contract strong bonds within a molecule is more costly. ${ }^{89}$ At around $75 \mathrm{GPa}\left(r_{s}=1.60\right)$, the shortest

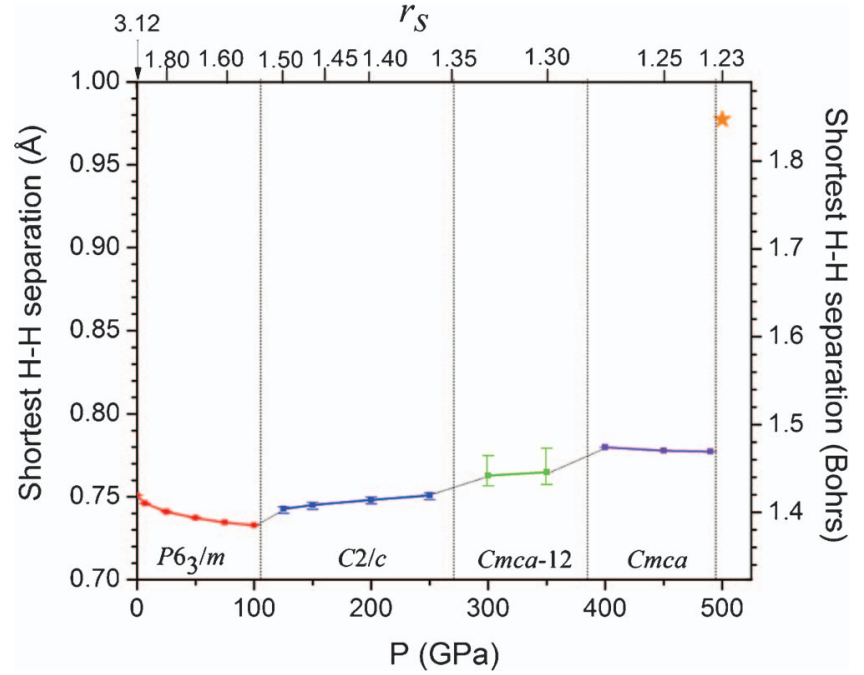

FIG. 7. Evolution of the average shortest $\mathrm{H}-\mathrm{H}$ distance (the intramolecular $\mathrm{H}-\mathrm{H}$ distance) under pressure. The maximal and minimal values are also indicated by a bar. The orange star indicates the shortest $\mathrm{H}-\mathrm{H}$ separation in the I4 ${ }_{1}$ /amd monatomic structures at $500 \mathrm{GPa}$.

distance between two $\mathrm{H}_{2}$ molecules is just half its value at $1 \mathrm{~atm}$. In the successive $\mathrm{C} 2 / \mathrm{c}, \mathrm{Cmca}-12$, and Cmca structures, this distance continues to decrease with pressure, reaching a value of $1.04 \AA$ at $490 \mathrm{GPa}$, whereas the shortest $\mathrm{H}-\mathrm{H}$ distance in the monatomic $I 4_{1} /$ amd structure is $0.98 \AA\left(1.85 a_{0}\right)$ at $500 \mathrm{GPa}$.

Note the general correspondence of this curve to Figure 2, a plot of the Wigner-Seitz radius $r_{s}$ vs $P$, taken from the equation of state for $\mathrm{H}_{2}$ of Caillabet et al. ${ }^{71}$

\section{B. When the intramolecular distance increases}

More interesting, perhaps, is the evolution of the intramolecular $\mathrm{H}-\mathrm{H}$ distance under pressure, because it is in some way a more sensitive probe of the short intermolecular interactions involved. And that intramolecular $\mathrm{H}-\mathrm{H}$ distance is an indication of the response of hydrogen to an increase of the electronic density, an essential point in the potential metallization of hydrogen by band overlap. In the following, the intramolecular $\mathrm{H}-\mathrm{H}$ distance is defined simply as the shortest separation between two protons, as sketched by the plain blue arrow in Figure 5.

In Figure 7 is plotted the evolution under pressure of the average intramolecular $\mathrm{H}-\mathrm{H}$ distance in the $P 6_{3} / m, C 2 / c$, Cmca-12, and Cmca unit cells. Since not all $\mathrm{H}_{2}$ molecules are equivalent, in some cases quite different intramolecular $\mathrm{H}-\mathrm{H}$ separations are involved but within the same structure. The bars in Figure 7 just indicate in each case the minimal and the maximal intramolecular $\mathrm{H}-\mathrm{H}$ distances in the structure considered. For comparison, once again the shortest $\mathrm{H}-$ $\mathrm{H}$ distance in the monatomic $\mathrm{I} 4_{1} /$ amd structure at $500 \mathrm{GPa}$ is indicated by an orange star.

In contrast to the evolution of the shortest intermolecular $\mathrm{H}-\mathrm{H}$ separations shown in Figure 6, the evolution of the intramolecular $\mathrm{H}-\mathrm{H}$ distance as a function of pressure is not smooth. As expected this is in part a consequence of the structural phase transitions encountered, but also of the 
relatively small range of the intramolecular $\mathrm{H}-\mathrm{H}$ separations. Indeed, whereas in our calculations the shortest intermolecular distance between two $\mathrm{H}_{2}$ molecules decreases from $3.1 \AA$ $\left(5.9 a_{0}\right)$ down to $1.0 \AA\left(1.9 a_{0}\right)$ between $1 \mathrm{~atm}$ and $490 \mathrm{GPa}$, the intramolecular $\mathrm{H}-\mathrm{H}$ distance varies in the range of $0.736 \AA\left(1.391 a_{0}\right)$ to $0.780 \AA\left(1.474 a_{0}\right)$, less than the estimated root mean amplitude of the intramolecular vibration due to the zero-point motion $\left(\sim 0.09 \AA\right.$ or $\left.0.17 a_{0}\right)$ and that of the libron displacements $\left(\sim 0.24 \AA\right.$ or $\left.0.46 a_{0}\right)$.

The general trend is clear; it is that the intramolecular $\mathrm{H}-\mathrm{H}$ distance does increase with pressure, but remains noticeably shorter than the shortest $\mathrm{H}-\mathrm{H}$ distance in the I $4_{1} /$ amd structure at $500 \mathrm{GPa}$. Nevertheless, different regimes can be identified before the transition to this monatomic structure. As the pressure increases in the domain of Phase $\mathrm{I}$, in a first regime, the intramolecular $\mathrm{H}-\mathrm{H}$ distance is shortened. With our computational methodology, it ranges from $0.751 \AA\left(1.420 a_{0}\right)$ at 1 atm to $0.733 \AA\left(1.386 a_{0}\right)$ at $100 \mathrm{GPa}$. This can be understood as a response to the increased repulsion between electrons of different $\mathrm{H}_{2}$ molecules, accompanying drastic reduction of the region where attractive van der Waals forces were operative. As mentioned earlier, such a shortening of the intramolecular $\mathrm{H}-\mathrm{H}$ distance has been observed experimentally by Loubeyre et $a l .^{45}$ and by Grazzi et al. ${ }^{46}$ by analyzing the roton bands in para $\mathrm{H}_{2}$, and it was also suggested by the increasing frequency of the Raman vibron in Phase I from 1 atm to $36 \mathrm{GPa} .{ }^{42}$ In the second paper of this series, we will discuss in detail the mechanism for this interesting contraction.

It should be noted that the experimental studies ${ }^{45,46}$ see a "turnover" in the $\mathrm{H}-\mathrm{H}$ intramolecular distance (as extracted from the roton spectra) from contracting under pressure to expanding, this occurring at $\sim 50 \mathrm{GPa}$ in Phase I (See Figure S7 in the supplementary material to this paper $^{73}$ for a graphical comparison of the experimental and calculated evolution of the intramolecular distance with pressure). In our calculations, this transition in the $P 6_{3} / m$ structure (which we relate to Phase I) does not take place until much higher pressures (see Paper III in our series (Ref. 10)).

In a second regime, from $100 \mathrm{GPa}$ to $400 \mathrm{GPa}$, the intramolecular $\mathrm{H}-\mathrm{H}$ distance increases with pressure. By analogy with its contraction (associated with a strengthening of the $\mathrm{H}_{2}$ bond), this increase of the intramolecular $\mathrm{H}-\mathrm{H}$ distance is proposed to be a consequence of the weakening of the $\mathrm{H}_{2}$ bond. The experimental measure here is the decrease of the vibron frequency whose origin is thought to be more "chemical" than "physical." If each hydrogen atom is to increase coordination under pressure, the $\mathrm{H}_{2}$ bond must weaken, and thus the intramolecular $\mathrm{H}-\mathrm{H}$ distance lengthens.

We will provide a more detailed orbital analysis of this phenomenon in the second and third papers of this series, ${ }^{9,10}$ but let us broach an explanation here. An isolated $\mathrm{H}_{2}$ molecule is characterized by a bonding $\sigma_{\mathrm{g}}$ orbital and an antibonding $\sigma_{\mathrm{u}} *$ orbital, as shown in Figure 8 .

Figure 9 shows schematically what happens at the orbital level when two $\mathrm{H}_{2}$ molecules interact. The mixing of $\sigma_{\mathrm{u}}{ }^{*}$ of one molecule into $\sigma_{\mathrm{g}}$ of the other results in electron transfer, partial depopulation of $\sigma_{\mathrm{g}}$ levels of both partners in the interaction, and (generally) population of their $\sigma_{\mathrm{u}} *$ antibonding

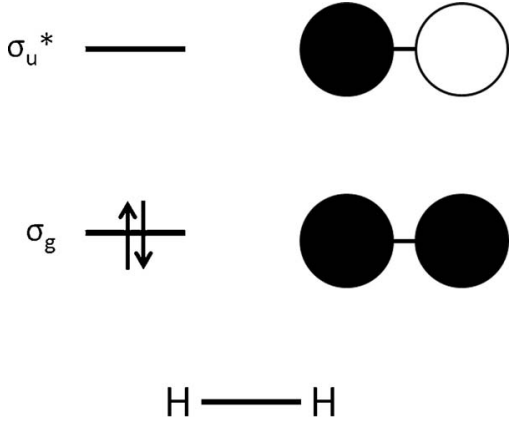

FIG. 8. Schematic highest occupied molecular orbital $(\mathrm{HOMO})-\sigma_{\mathrm{g}}-$ and lowest unoccupied molecular orbital (LUMO) $-\sigma_{\mathrm{u}} *-$ of an isolated $\mathrm{H}_{2}$ molecule. Black and white regions above simply indicate relative phases of the wave function; no implications are being inferred of the coefficient amplitudes.

molecular orbitals. An inevitable result of $\mathrm{H}_{2}$ compression is therefore electron transfer out of the $\sigma_{\mathrm{g}}$ and into the $\sigma_{\mathrm{u}}{ }^{*}$.

A quite similar phenomenon has been noted in the remarkable organometallic complexes where an $\mathrm{H}_{2}$ molecule is bonded side-on to a transition metal center. The "other molecule" is then an organometallic fragment. ${ }^{90}$ These structures, in which the intramolecular $\mathrm{H}-\mathrm{H}$ separation is remarkably elongated relative to an isolated $\mathrm{H}_{2}$ molecule, will be discussed in detail in the second paper of this series as a useful model for the physical and chemical mechanisms at work in dense hydrogen under pressure. ${ }^{9}$ The electron transfers out of the $\sigma_{\mathrm{g}}$ and into the $\sigma_{\mathrm{u}}{ }^{*}$ which both weaken the $\mathrm{H}-\mathrm{H}$ bond, need not be balanced in magnitude; i.e., if symmetry allows it, a $\mathrm{H}_{2}$ unit may depart from neutrality and be negatively or positively charged.

In a third regime, at very high pressures, say from $400 \mathrm{GPa}$ to $490 \mathrm{GPa}$ (the average shortest intermolecular separation is then less than $1.1 \AA$ or $2.08 a_{0}$ ), the intramolecular $\mathrm{H}-\mathrm{H}$ distance slightly decreases. This probably marks an entry into the regime in which covalent bonds are compressed.

Now to summarize what we intend to render plausible through further analysis in the next papers in the series: The effective intramolecular $\mathrm{H}-\mathrm{H}$ distance observed in dense hydrogen under pressure thus results from two effects that work in opposing directions:

1 An increase of the "physical" repulsion between the $\mathrm{H}_{2}$ molecules (in a way building a wall around a molecule) tends to shorten and strengthen the $\mathrm{H}_{2}$ bond,

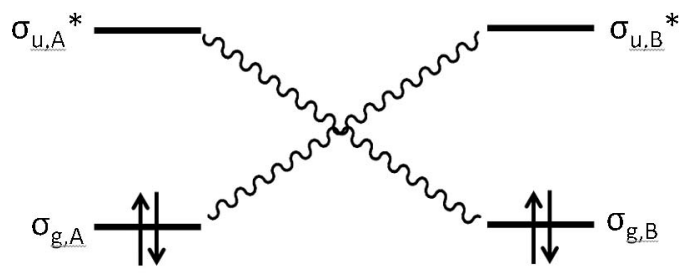

Molecule A

Molecule B

FIG. 9. Schematic representation of interaction between the molecular orbitals of two $\mathrm{H}_{2}$ molecules. The wiggly lines represent interactions between the orbitals specified. 
2 An increase of the "chemical" orbital interactiondepopulation of $\sigma_{\mathrm{g}}$, population of $\sigma_{\mathrm{u}}{ }^{*}$-tends to lengthen and soften the $\mathrm{H}_{2}$ bond.

Between $1 \mathrm{~atm}\left(r_{s}=3.12\right)$ and $100 \mathrm{GPa}\left(r_{s}=1.54\right)$ - the average shortest intermolecular $\mathrm{H}-\mathrm{H}$ separation is then situated between $3.1 \AA\left(5.9 a_{0}\right)$ and $1.6 \AA\left(3.1 a_{0}\right)$-we believe that the intramolecular distances are governed by the repulsive interactions between $\mathrm{H}_{2}$ molecules. In a second regime, between $100 \mathrm{GPa}\left(r_{s}=1.54\right)$ and $400 \mathrm{GPa}\left(r_{s}=1.28\right)$, a pressure range in which the average shortest intermolecular separation is comprised between $1.6 \AA\left(3.1 a_{0}\right)$ and $1.1 \AA(2.0$ $\left.a_{0}\right)$, orbital interactions are dominant. And in a third regime, above $400 \mathrm{GPa}$, bond compression occurs again by a repulsive mechanism. The reasons for the evolution of the intramolecular $\mathrm{H}-\mathrm{H}$ distance will be studied in more detail in the second paper of this series. ${ }^{9}$ There, we will develop further the arguments evoked here, and also support them by numerical experiments.

\section{Does hydrogen go towards equalization of its $\mathrm{H}-\mathrm{H}$ distances?}

Let us return to the question of major interest in this deceptively simple system, namely the occurrence of $\mathrm{H}-\mathrm{H}$ distance equalization or its absence, in so far as the Pickard and Needs calculations may reveal it. Because of the evolution of the intermolecular $\mathrm{H}-\mathrm{H}$ separation which decreases with pressure (Figure 6), and that of the intramolecular $\mathrm{H}-\mathrm{H}$ distance, which globally increases with pressure (Figure 7), the shortest and second shortest $\mathrm{H}-\mathrm{H}$ distances in solid hydrogen evolve towards equalization with pressure. This evolution can be measured concisely, if not uniquely, by introducing the following equalization function:

$$
\xi(P)=1-\frac{R_{\mathrm{H}_{2}-\mathrm{H}_{2}}(P)-r_{\mathrm{H}-\mathrm{H}}(P)}{R_{\mathrm{H}_{2}-\mathrm{H}_{2}}\left(P_{1 \mathrm{~atm}}\right)-r_{\mathrm{H}-\mathrm{H}}\left(P_{1 \mathrm{~atm}}\right)} .
$$

Here $r_{\mathrm{H}-\mathrm{H}}(P)$ and $R_{\mathrm{H}_{2}-\mathrm{H}_{2}}(P)$ are the shortest and second shortest $\mathrm{H}-\mathrm{H}$ separations at pressure $P$, respectively, and $r_{\mathrm{H}-\mathrm{H}}\left(P_{1 \mathrm{~atm}}\right)$ and $R_{\mathrm{H}_{2}-\mathrm{H}_{2}}\left(P_{1 \text { atm }}\right)$ the shortest and second shortest $\mathrm{H}-\mathrm{H}$ separations at $P=1 \mathrm{~atm}$ (these are $0.751 \AA$ $\left(1.420 a_{0}\right)$ and $3.105 \AA\left(5.870 a_{0}\right)$, respectively, with our computational methodology). Note that all separations are taken as time average values. This equalization function, $\xi(P)$, is thus defined in such a way that it takes on values between 0 and 1 . By definition, $\xi(P)$ is equal to 0 at 1 atm and tends towards 1 when the intramolecular and shortest intermolecular $\mathrm{H}-\mathrm{H}$ separations become equal. The evolution of $\xi$ over the entire pressure range of the Pickard and Needs phases is now plotted in Figure 10.

Because of the strong volume compression from 1 atm to $100 \mathrm{GPa}$ (corresponding to the progressive collapse of the van der Waals region of interactions), $\xi$ increases dramatically in this pressure regime. It then increases more softly with pressure and seems to be reaching a plateau. At $490 \mathrm{GPa}$, it is still notably below the value of 0.9 .

The fact that the $I 4_{1} /$ amd monatomic $\xi=1$ structure appears to become the most stable structure around $490 \mathrm{GPa}$, where the equalization function for other structures is still be-

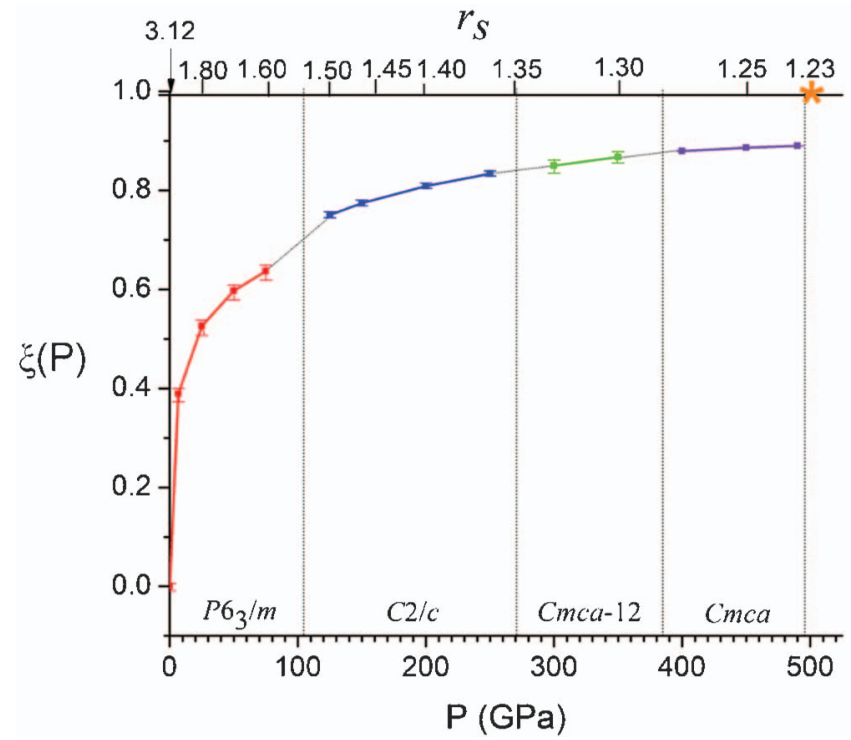

FIG. 10. Evolution of the equalization function (Eq. (6)) with pressure in dense hydrogen. The orange star indicates that the monatomic $I 4_{1} /$ amd structure is characterized by perfect equalization of the $\mathrm{H}-\mathrm{H}$ separations.

low 0.9 , could suggest that dense hydrogen will eventually reach a monatomic phase via a phase transition involving a drastic dissociation of the $\mathrm{H}_{2}$ pairs, rather than continuously. We will see in the second ${ }^{9}$ and third ${ }^{10}$ papers of this series that the process could indeed be more complex.

It is remarkable that at $P=300 \mathrm{GPa}$, where we know that $\mathrm{H}_{2}$ molecules are present from the persistence of the $\mathrm{H}-\mathrm{H}$ vibron band in the Raman spectrum of dense hydrogen, the degree of equalization of the shortest and second shortest $\mathrm{H}-\mathrm{H}$ separations is already $85 \%$, the shortest intermolecular $\mathrm{H}-\mathrm{H}$ separation being $1.12 \AA$, i.e., only 1.5 times the intramolecular $\mathrm{H}-\mathrm{H}$ separation $-0.76 \AA$.

We also know from the work of Pickard and Needs ${ }^{6}$ and of Johnson and Ashcroft ${ }^{57}$ that the $\mathrm{C} / \mathrm{c}, \mathrm{Cmca}-12$, and Cmca structures with paired hydrogens are all predicted to be metallic by band overlap above around 410GPa or $r_{s}=1.27$ (taking into account the fact that DFT methods underestimate band gaps). Note that at this pressure, $\xi \approx 0.88$. So it appears that one can indeed attain a metallic state of hydrogen without it being an "atomic crystal." In other words, our analysis supports the contention that hydrogen is likely to become metallic before it becomes a monatomic crystal.

\section{THE BEGINNINGS OF A CONCLUSION}

A definitive summary of our analysis of the physical and chemical development of dense hydrogen and its structures will be found in the third and fourth paper in this series. What we have done here, in the first paper, is to look at the structures predicted by Pickard and Needs to be the most stable static ground state arrangements for solid hydrogen at $0 \mathrm{~K}$ under pressure, and to use them as a kind of laboratory for learning more about hydrogen under pressure. Our initial focus was on the evolution of the intramolecular and shortest intermolecular $\mathrm{H}-\mathrm{H}$ distances, to determine to what extent solid 
hydrogen undergoes an equalization of its $\mathrm{H}-\mathrm{H}$ distances under pressure.

Following the most stable structure of solid hydrogen between $1 \mathrm{~atm}\left(r_{s}=3.12\right)$ and $500 \mathrm{GPa}\left(r_{s}=1.23\right)$, the shortest intermolecular $\mathrm{H}-\mathrm{H}$ separation decreases with pressure whereas the intramolecular $\mathrm{H}-\mathrm{H}$ distance globally increases, in agreement with general intuition. Nevertheless, hydrogen seems to resist perfect equalization of its $\mathrm{H}-\mathrm{H}$ distance, reinforcing the idea of a possible molecular metallic state of solid hydrogen, before its dissociation.

In agreement with the experimental evidence, the evolution of the intramolecular $\mathrm{H}-\mathrm{H}$ distance passes through three several regimes. At lower pressures, it decreases slightly with pressure, then as the pressure is increased the intramolecular $\mathrm{H}-\mathrm{H}$ distance elongates. And at still higher pressures, it declines again. In the second paper of this series, we will present in detail some molecular models to help us delineate the physical and chemical mechanisms at work in intramolecular $\mathrm{H}-\mathrm{H}$ distance changes as pressure is applied. ${ }^{9}$

One final comment: We have proposed an equalization function that may be useful in looking at the evolution of $\mathrm{H}-\mathrm{H}$ separations obtained from static calculations as one approaches metallization. There are certainly other ways to study the approach to metallization-in particular molecular dynamics of the Car-Parrinello kind, path-integral Monte Carlo and related methods. We suggest that the equalization function will be just as useful in describing the simulated structure of hydrogen solid or liquid in those cases. For there will be a range of nearest and next nearest $\mathrm{H}-\mathrm{H}$ separations in these calculations, and the spreads of these will then be a measure of fluctuations in the material, and will be a function of temperature. Moreover, we think that this function may be a useful tool to compare quantitatively the behavior of hydrogen by itself under pressure and that of hydrides, in the context of testing the idea of "chemical pre-compression" introduced a few years ago by Ashcroft. ${ }^{91}$

We welcome the reader to the remaining papers in this series. In the second paper existing and new molecular models are used to isolate two effects - one physical, one orbital or chemical - on the intra- and intermolecular $\mathrm{H}-\mathrm{H}$ separations as the pressure increases.

\section{ACKNOWLEDGMENTS}

We thank Paul Loubeyre and Isaac Silvera for providing us with important information. Our work was supported by EFree, an Energy Frontier Research Center funded by the U.S. Department of Energy (DOE), Office of Science, Office of Basic Energy Sciences under Award Number DESC0001057 at Cornell. The work was also funded by the National Science Foundation (NSF) through CHE-0910623 and Grant No. DMR-0907425. V.L. would like to thank the FrancoAmerican Commission for Educational Exchange for its financial support. Calculations were performed in part at the Cornell NanoScale Facility, a member of the National Nanotechnology Infrastructure Network, which is supported by the NSF (Grant No. ECS-0335765). This research was also supported by the NSF through TeraGrid resources provided by NCSA.
${ }^{1}$ T. Kato, Trans. Amer. Math. Soc. 70, 195 (1951).

${ }^{2}$ T. Kato, Commun. Pure. Appl. Math. 10, 151 (1957).

${ }^{3}$ H. Bethe, Z. Phys. 57, 815 (1929).

${ }^{4}$ R. N. Hill, Phys. Rev. Lett. 38, 643 (1977).

${ }^{5}$ Note that the spherically symmetric ground rotational state $J=0$ couples exclusively with the para nuclear spin state $(I=0)$ of the $\mathrm{H}_{2}$ molecules. Thus, at ambient pressure and at the limit $T=0 \mathrm{~K}$, the thermodynamic equilibrium corresponds to $100 \% p-\mathrm{H}_{2}$ molecules. The lowest rotational state to which the ortho nuclear spin state can be coupled is $J=1$. At one atmosphere, normal hydrogen is composed at $25 \%$ of para-hydrogen and at $75 \%$ of ortho-hydrogen. The typical energy gap between $J=0$ and $J=2$ rotational states is about $44 \mathrm{meV}$ (or $355 \mathrm{~cm}^{-1}$ ).

${ }^{6}$ C. J. Pickard and R. J. Needs, Nat. Phys. 3, 473 (2007).

${ }^{7}$ C. J. Pickard and R. J. Needs, $\Psi_{\mathrm{k}}$ Newsletter 100, 42 (2010).

${ }^{8}$ C. J. Pickard and R. J. Needs, J. Phys.: Condens. Matter 23, 053201 (2011).

${ }^{9}$ V. Labet, R. Hoffmann, and N. W. Ashcroft, J. Chem. Phys. 136, 074502 (2012).

${ }^{10}$ V. Labet, R. Hoffmann, and N. W. Ashcroft, J. Chem. Phys. 136, 074503 (2012).

${ }^{11}$ V. Labet, R. Hoffmann, and N. W. Ashcroft, J. Chem. Phys. 136, 074504 (2012).

${ }^{12}$ C. A. Coulson, Rev. Mod. Phys. 32, 170 (1960).

${ }^{13}$ R. F. W. Bader, Chem. Rev. 91, 893 (1991).

${ }^{14}$ B. Silvi and A. Savin, Nature (London) 371, 683 (1994).

${ }^{15}$ A. E. Reed, L. A. Curtiss, and F. Weinhold, Chem. Rev. 88, 899 (1988).

${ }^{16}$ K. Kitaura and K. Morokuma, Int. J. Quantum Chem. 10, 325 (1976).

${ }^{17}$ J. Feng, R. Hoffmann, and N. W. Ashcroft, J. Chem. Phys. 132, 114106 (2010).

${ }^{18}$ R. F. Berger, P. L. Walters, S. Lee, and R. Hoffmann, Chem. Rev. 111, 4522 (2011).

${ }^{19}$ E. Wigner and H. B. Huntington, J. Chem. Phys. 3, 764 (1935).

${ }^{20}$ S. T. Weir, A. C. Mitchell, and W. J. Nellis, Phys. Rev. Lett. 76, 1860 (1996).

${ }^{21}$ P. Loubeyre, F. Occelli, and R. LeToullec, Nature (London) 46, 613 (2002).

${ }^{22}$ A. F. Goncharov, E. Gregoryanz, R. J. Hemley, and H. K. Mao, Proc. Natl. Acad. Sci. U.S.A. 98, 14234 (2001).

${ }^{23}$ C. Narayana, H. Luo, J. Orloff, and A. L. Ruloff, Nature (London) 393, 46 (1998).

${ }^{24}$ P. P. Edwards and F. Hensel, Nature (London) 388, 621 (1997).

${ }^{25}$ M. I. Eremets and I. A. Troyan, Nature Mater. 10, 927 (2011); see, however, W. J. Nellis, A. L. Ruoff, and I. F. Silvera, e-print arXiv:1201.0407.

${ }^{26}$ H. K. Mao and R. J. Hemley, Rev. Mod. Phys. 66, 671 (1994).

${ }^{27}$ I. F. Silvera, Rev. Mod. Phys. 52, 393 (1980).

${ }^{28}$ K. Inoue, H. Kanzaki, and S. Suga, Solid State Commun. 30, 627 (1979).

${ }^{29}$ N. W. Ashcroft, Phys. Rev. Lett. 21, 1748 (1968).

${ }^{30}$ E. Babaev, A. Sudbø, and N. W. Ashcroft, Nature (London) 431, 666 (2004).

${ }^{31}$ M. A. Morales, C. Pierleoni, E. Schwegler, and D. M. Ceperley, Proc. Natl. Acad. Sci. U.S.A. 107, 12799 (2010).

${ }^{32}$ I. Silvera, Proc. Natl. Acad. Sci. U.S.A. 107, 12743 (2010).

${ }^{33}$ R. J. Hemley, High Press. Res. 30, 581 (2010).

${ }^{34}$ F. Datchi, P. Loubeyre, and R. LeToullec, Phys. Rev. B 61, 6535 (2000).

${ }^{35}$ E. Gregoryanz, A. F. Goncharov, K. Matsuishi, H. K. Mao, and R. J. Hemley, Phys. Rev. Lett. 90, 175701 (2003).

${ }^{36}$ N. Subramanian, A. F. Goncharov, V. V. Struzhkin, M. Somayazulu, and R. J. Hemley, Proc. Natl. Acad. Sci. U.S.A. 108, 6014 (2011).

${ }^{37}$ S. Deemyad and I. F. Silvera, Phys. Rev. Lett. 100, 155701 (2008).

${ }^{38}$ M. I. Eremets and I. A. Trojan, JETP Lett. 89, 174 (2009).

${ }^{39}$ S. A. Bonev, E. Schwegler, T. Ogitsu, and G. Galli, Nature (London) 421, 669 (2004).

${ }^{40}$ P. Loubeyre, R. LeToullec, D. Hausermann, M. Hanfland, R. J. Hemley, H. K. Mao, and L. W. Finger, Nature (London) 383, 702 (1996).

${ }^{41}$ M. Hanfland, R. J. Hemley, and H. K. Mao, Phys. Rev. Lett. 70, 3760 (1993).

${ }^{42}$ S. K. Sharma, H. K. Mao, and P. M. Bell, Phys. Rev. Lett. 44, 886 (1980).

${ }^{43}$ S. K. Sharma, H. K. Mao, and P. M. Bell, Phys. Rev. Lett. 46, 1109 (1981).

${ }^{44}$ I. F. Silvera and R. J. Wijngaarden, Phys. Rev. Lett. 47, 39 (1981).

${ }^{45}$ P. Loubeyre, M. Jean-Louis, and I. F. Silvera, Phys. Rev. B 43, 10191 (1991).

${ }^{46}$ F. Grazzi, M. Moraldi, and L. Ulivi, Europhys. Lett. 68, 664 (2004)

${ }^{47}$ R. J. Hemley and H. K. Mao, Phys. Rev. Lett. 61, 857 (1988).

${ }^{48}$ H. E. Lorenzana, I. F. Silvera, and K. A. Goettel, Phys. Rev. Lett. 63, 2080 (1989). 
${ }^{49}$ Y. Akahama, M. Nishimura, H. Kawamura, N. Hirao, Y. Ohishi, and K. Takemura, Phys. Rev. B 82, 060101(R) (2010).

${ }^{50}$ R. G. Hemley, Z. G. Soos, M. Hanfland, and H. K. Mao, Nature (London) 369, 383 (1994).

${ }^{51}$ I. I. Mazin, R. J. Hemley, A. F. Goncharov, M. Hanfland, and H. K. Mao, Phys. Rev. Lett. 78, 1066 (1997).

${ }^{52}$ P. Loubeyre, R. LeToullec, D. Hausermann, M. Hanfland, R. J. Hemley, H. K. Mao, and L. W. Finger, Nature (London) 383, 702 (1996).

${ }^{53}$ D. E. Ramaker, L. Kumar, and F. E. Harris, Phys. Rev. Lett. 34, 812 (1975).

${ }^{54}$ C. Friedli and N. W. Ashcroft, Phys. Rev. B 16, 662 (1977).

${ }^{55}$ N. W. Ashcroft, in Molecular Systems Under High Pressure: Proceedings of the II Archimedes Workshop on Molecular Systems Under Pressure, Catania, Italy, 28-31 May 1990, edited by R. Pucci and G. Piccitto (Elsevier, Amsterdam, 1991), pp. 201-222.

${ }^{56}$ C. F. Richardson and N. W. Ashcroft, Phys. Rev. Lett. 78, 118 (1997).

${ }^{57}$ K. A. Johnson and N. W. Ashcroft, Nature (London) 403, 632 (2000).

${ }^{58}$ V. E. Fortov, R. I. Ilkaev, V. A. Arinin, V. V. Burtzev, V. A. Golubev, I. L. Iosilevskiy, V. V. Khrustalev, A. L. Mikhailov, M. A. Mochalov, V. Ya. Ternovoi, and M. V. Zhernokletov, Phys. Rev. Lett. 99, 185001 (2007).

${ }^{59}$ K. Moulopoulos and N. W. Ashcroft, Phys. Rev. B 41, 6500 (1990).

${ }^{60}$ T. W. BarbeeIII, A. García, M. L. Cohen, and J. L. Martins, Phys. Rev. Lett. 62, 1150 (1989).

${ }^{61}$ E. Kaxiras, J. Broughton, and R. J. Hemley, Phys. Rev. Lett. 67, 1138 (1991).

${ }^{62}$ K. Nagao, H. Nagara, and S. Matsubara, Phys. Rev. B 56, 2295 (1997).

${ }^{63}$ B. Edwards, N. W. Ashcroft, and T. Lenosky, Europhys. Lett. 34, 519 (1996).

${ }^{64}$ J. Kohanoff, S. Scandolo, G. L. Chiarotti, and E. Tossatti, Phys. Rev. Lett. 78, 2783 (1997).

${ }^{65}$ H. Kitamura, S. Tsuneyuki, T. Ogitsu and T. Miyake, Nature (London) 404, 259 (2000).

${ }^{66}$ D. M. Ceperley and B. J. Alder, Phys. Rev. B 36, 2092 (1987).

${ }^{67}$ V. Natoli, R. M. Martin, and D. M. Ceperley, Phys. Rev. Lett. 70, 1952 (1993).

${ }^{68}$ V. Natoli, R. M. Martin, and D. M. Ceperley, Phys. Rev. Lett. 74, 1601 (1995).

${ }^{69}$ J. M. McMahon and D. M. Ceperley, Phys. Rev. Lett. 106, 165302 (2011).
${ }^{70} \mathrm{H}$. Liu, H. Wang, and Y. Ma, e-print arXiv:1112.1174.

${ }^{71}$ L. Caillabet, S. Mazevet, and P. Loubeyre, Phys. Rev. B 83, 094101 (2011).

${ }^{72}$ To calculate the relation between $P$ and $r_{s}$ at $T=0 \mathrm{~K}$, only the contribution from the electronic ground-state energy was taken into account. The contribution from the zero-point motion, which can be calculated from the equation of state provided by Caillabet et al. (Ref. 71) was neglected.

${ }^{73}$ See supplementary material http://dx.doi.org/10.1063/1.3679662 for relevant figures.

${ }^{74}$ C. J. Pickard and R. J. Needs, Phys. Status Solidi B 246, 536 (2009).

${ }^{75}$ J. S. Tsé, D. D. Klug, Y. Yao, Y. L. Page, and J. R. Rodgers, Solid State Commun. 145, 5 (2008).

${ }^{76}$ D. M. Ceperley and M. H. Kalos, in Monte Carlo Methods in Statistical Physics, edited by K. Binder (Springer-Verlag, Berlin, 1979), p. 145.

${ }^{77}$ D. Bohm and T. Staver, Phys. Rev. 84, 836 (1951).

${ }^{78}$ H. R. Glyde, Excitations in Liquid and Solid Helium (Clarendon, Oxford, 1995).

${ }^{79}$ From the work done by Takezawa et al. on the effect of the zero-point energy of the proton motions on the pressure of molecular dissociation in dense hydrogen (T. Takezawa, K. Nagao, and H. Nagara, J. Low Temp. Phys. 123, 315 (2001)), it can be anticipated that in the work done by Pickard and Needs, the pressure corresponding to the transition from the Cmca structure to the $I 4_{1} /$ amd one is overestimated.

${ }^{80}$ G. Kresse and J. Hafner, Phys. Rev. B 47, 558 (1993).

${ }^{81}$ G. Kresse and J. Furthmüller,Comput. Mater. Sci. 6, 15 (1996).

${ }^{82}$ G. Kresse and J. Furthmüller, Phys. Rev. B 54, 11169 (1996).

${ }^{83}$ J. P. Perdew, K. Burke, and M. Ernzerhof, Phys. Rev. Lett. 77, 3865 (1996).

${ }^{84}$ J. P. Perdew, K. Burke, and M. Ernzerhof, Phys. Rev. Lett. 78, 1396 (1997).

${ }^{85}$ P. E. Bloch, Phys. Rev. B 50, 17953 (1994).

${ }^{86}$ G. Kresse and D. Joubert, Phys. Rev. B 59, 1758 (1999).

${ }^{87}$ H. J. Monkhorst and J. D. Pack, Phys. Rev. B 13, 5188 (1976).

${ }^{88}$ In their work, Pickard and Needs (Ref. 4) used AIRSS coupled with the CASTEP plane-wave code, the Perdew-Burke-Ernzerhof generalized gradient approximation density functional, and ultrasoft pseudopotentials.

${ }^{89}$ W. Grochala, R. Hoffmann, J. Feng, and N. W. Ashcroft, Angew. Chem., Int. Ed. 46, 3620 (2007).

${ }^{90}$ G. J. Kubas, Chem. Rev. 107, 4152 (2007).

${ }^{91}$ N. W. Ashcroft, Phys. Rev. Lett. 92, 187002 (2004). 